\title{
Barotropic Instability across the Moat and Inner Eyewall Dissipation: A Numerical Study of Hurricane Wilma (2005)
}

\author{
TsZ-Kin Lai, Konstantinos Menelaou, And M. K. Yau \\ Department of Atmospheric and Oceanic Sciences, McGill University, Montreal, Quebec, Canada
}

(Manuscript received 10 July 2018, in final form 8 January 2019)

\begin{abstract}
Radar imagery of some double-eyewall tropical cyclones shows that the inner eyewalls become elliptical prior to their dissipation. These elliptical features indicate that the barotropic instability (BI) across the moat (aka, type-2 BI) may play a role in the process. To investigate the mechanism for dissipation, a WRF simulation of Hurricane Wilma (2005) is performed. The results reveal an elliptical elongation of the inner eyewall and a change in the structure of the radial flow from wavenumber (WN) 1 to WN 2 at the lower levels. A linear stability analysis as well as idealized nonlinear experiments using a nondivergent barotropic vorticity model initialized with the vorticity fields before the change in the dominant wavenumber of the radial flow are presented with the results supporting the presence of a type-2 BI at the lower levels. The accompanying $\mathrm{WN}-2$ radial flow is also found to dilute the vorticity within the inner eyewall and the eye. However, this dilution is not seen at higher levels as the type-2 BI becomes weak and short lived at the middle levels and reaches its weakest strength at the upper levels. This phenomenon is traced to the fact that a higher growth rate comes with a narrower moat for type-2 BI. As the outward slope of the outer eyewall is larger than that of the inner eyewall, the moat width increases with height so that the growth rate decreases with height. The results presented here thus highlight the potential role played by the barotropic instability across the moat in inner eyewall dissipation.
\end{abstract}

\section{Introduction}

It is well known that secondary eyewall formation (SEF) is a common feature occurring during the mature stage of a tropical cyclone (TC). In SEF, a new eyewall forms outside the existing eyewall and an eyewall replacement cycle (ERC) often follows thereafter. During an ERC the secondary (or outer) eyewall gradually contracts while the inner eyewall weakens and eventually dissipates. Over the years, with the advances in satellite and radar observations as well as numerical modeling, double eyewalls and the corresponding ERCs have received significant attention (e.g., Willoughby et al. 1982; Black and Willoughby 1992; Houze et al. 2006, 2007; Sitkowski et al. 2011; Yang et al. 2013). In general, it has been revealed that an ERC usually halts the intensification of the TC, broadens the area of damaging gale-force winds, and even leads to TC weakening followed possibly by a period of reintensification. Subsequent to the replacement of the inner eyewall by the outer eyewall, another SEF and ERC could occur

\footnotetext{
Corresponding author: Tsz-Kin Lai, eric.lai@mail.mcgill.ca
}

(Hawkins and Helveston 2008). Furthermore, the phenomenon of tertiary eyewall (although more rarely) has also been reported in the literature (e.g., McNoldy 2004; Zhao et al. 2016).

Despite the large number of papers on both SEF and ERCs in the past few decades, most of the published work focused on the fundamental mechanisms responsible for their initial formation. The proposed SEF mechanisms include vortex Rossby wave-mean flow interaction (e.g., Montgomery and Kallenbach 1997; Martinez et al. 2011; Menelaou et al. 2012, 2013), barotropic vorticity dynamics (Kuo et al. 2004, 2008), $\beta$-skirt axisymmetrization (Terwey and Montgomery 2008), dynamics within the atmospheric boundary layer (ABL) (e.g., Huang et al. 2012; Abarca and Montgomery 2013, 2014; Kepert 2013; Montgomery et al. 2014; Kepert and Nolan 2014; Wang et al. 2016), and the dynamics of mesoscale and convective-scale rainbands (e.g., Judt and Chen 2010; Rozoff et al. 2012; Zhu and Zhu 2014).

On the other hand, there are relatively few studies on the mechanism responsible for the dissipation of the inner eyewall (the completion of an ERC) and the subject remains not well understood. In some early 
studies, Willoughby et al. (1982) and Willoughby (1988) suggested that the outflow associated with the inner eyewall is counteracted by the forced upper-level inflow and descent from the outer eyewall leading to a disruption of the secondary circulation. However, this hypothesis was not supported by the findings of Rozoff et al. (2008) as the latter results (obtained by using the transverse circulation equation associated with a balanced vortex model) indicated that the decay of deep convection in the inner eyewall arises from a rapid increase of inner-core static stability resulting from the strengthening outer eyewall convection.

In a different context, another dissipation mechanism suggests that the development of an outer eyewall actively contributes to the inner eyewall demise by interrupting its low-level inflow (e.g., Samsury and Zipser 1995; Houze et al. 2007). This imposed barrier can have a twofold effect. First, it acts to reduce the boundary layer inflow and convergence underneath and just outside the inner eyewall. Such reduction decreases the inward transfer of angular momentum, which is a necessary ingredient for the maintenance of the inner eyewall against surface friction (Huang et al. 2012). ${ }^{1}$ Second, by suppressing the moist entropy-rich inflowing air, the barrier reduces the supply of moist entropy to the inner eyewall. As a result, the warm core of the inner eyewall cannot be maintained. According to the wind-induced surface heat exchange mechanism (WISHE; Emanuel 1986), a weaker warm core leads to weaker inflow, which in turn further weakens the warm core and so on. Zhou and Wang (2011) performed an axisymmetric equivalent potential temperature $\theta_{e}$ budget analysis using the output from their three-dimensional, nonhydrostatic, and full-physics numerical experiment. They found that when the two eyewalls are close, there is a strong cold $\theta_{e}$ advection to the inner eyewall within the boundary layer. As a result, the inner eyewall abruptly dissipates because the high moist entropy column can no longer be maintained. Of interest, these results seem to link the width of the moat (an annular region separating the two eyewalls) to the degree to which the outer eyewall impacts (negatively) the inner eyewall, and also link the time for completion of an ERC to the contraction rate of the outer eyewall.

Besides the direct suppression of moist entropy supply due to the barrier effect, the moist entropy supply can

\footnotetext{
${ }^{1}$ Here, it should be noted that different to Huang et al. (2012), Kepert (2013) argued that SEF tends to increase the frictionally induced secondary circulation. If the moat separating the two eyewalls is characterized by a local vorticity maximum, then sufficient subsidence may occur there to maintain the inner eyewall's updraft. Readers with interest on this debate are also referred to Montgomery et al. (2014) and Kepert and Nolan (2014) for further details.
}

also be indirectly reduced by downdrafts (linked to latent heat release in secondary eyewalls) within the moat region. These downdrafts can transport low $\theta_{e}$ from the middle levels to the boundary layer inflow region (e.g., Barnes et al. 1983) and thereby act to decrease convection and high vorticity in the inner eyewall. However, this indirect mechanism is not supported by the findings of Zhou and Wang (2011).

It should be noted, that the mechanisms described above are all axisymmetric. In the most recent decade, more attention has been directed to the asymmetric aspect of ERCs. Didlake et al. (2017) analyzed observation obtained during the ERCs of Hurricane Gonzalo and found evidence of descent from the outer eyewall overriding the updraft of the decaying inner eyewall in the downshear quadrants. The inference is that the aforementioned suppression mechanism of inner eyewall outflow suggested by Willoughby et al. (1982) and Willoughby (1988) could work in concert with the decreasing moist entropy supply to weaken the inner eyewall. However, Tsujino et al. (2017) showed from their simulation of Typhoon Bolaven (2012) that the asymmetric structure of the outer eyewall had a significant impact on the contraction rate of the outer eyewall. Moreover, their moist entropy budget and backward trajectory analyses indicated that the inward moist entropy supply within the boundary layer was still sufficient to maintain the long-lived inner eyewall (maintained for more than $20 \mathrm{~h}$; defined by Yang et al. 2013) of the simulated Bolaven. This indicates that boundary layer effects could play an important role in the inner eyewall dissipation. A similar finding based on the observational analysis of Hurricane Ike (2008) was reported by Zhang and Perrie (2018). Ike's double eyewalls were maintained for more than $30 \mathrm{~h}$.

Barotropic instabilities have been found to be associated with the double-eyewall phenomenon. Kossin et al. (2000, hereafter KSM00) identified two types of barotropic instability in the vorticity field of TCs with a double-eyewall structure. The first is the instability across the outer eyewall (referred to as type- 1 instability) and the second is the instability across the moat (referred to as type-2 instability). These instabilities can yield asymmetric features in the evolution of the vorticity field. For example, in a composite study of TCs having multiple eyewalls ${ }^{2}$ in the western North Pacific basin

\footnotetext{
${ }^{2}$ Although "concentric eyewalls" is widely used in the literature, it is believed that this terminology can be rather misleading especially when the inner eyewall is in trochoidal oscillation. Therefore, the phrase "multiple eyewalls" is preferred in this paper. It refers to any two or more coexisting eyewalls of a TC without the restriction that the geometric centers of all the inner and outer eyewalls must be the same.
} 
from 1997 to 2011, Yang et al. (2013) conjectured that barotropic instabilities might play a role in determining the maintenance time of the multiple-eyewall structures. However, their conjecture is based on the statistics of the observed size of the outer eyewall and the width of the moat. They provided no direct evidence for the presence of the instabilities nor a detailed study of the underlying dynamics. Nevertheless, radar imagery of some TCs with double eyewalls (McNoldy 2012) did show asymmetric features in the inner eyewalls (especially elliptical shapes) for a period of time prior to their dissipation [e.g., Hurricane Earl (2010) ${ }^{3}$ and Hurricane Maria $\left.(2017)^{4}\right]$. Since the elliptical evolution could in principle be explained by the type- 2 barotropic instability proposed by KSM00, the observed features from the radar imagery may imply a possible role played by the instability in inner eyewall dissipation. It is one of the objectives of the present study to validate or refute such a hypothesis.

Specifically, the aim of our study is to provide insight on the fundamental mechanisms responsible for inner eyewall dissipation. We attempt to determine whether barotropic instability that operates across the moat region is a suitable candidate that can partly explain the inner eyewall dissipation. In this first part of the study, the analysis will be based on a numerical investigation of Hurricane Wilma (2005). Wilma was the most intense hurricane recorded in the Atlantic basin, and it had two ERCs with the first one lasting for about $24 \mathrm{~h}$ (Chen et al. 2011). Our focus will be on the first ERC of Wilma.

The next section presents a brief overview of Hurricane Wilma. Section 3 provides the configurations of the numerical model and the simulation. Section 4 contains a description of some simulated inner-core structures and their evolution during Wilma's first ERC. Section 5 discusses these results with particular focus on the relation between barotropic instability across the moat and the dissipation of the inner eyewall using the tools of linear stability analysis and nonlinear numerical experiments with a $2 \mathrm{D}$ nondivergent barotropic vorticity model. The conclusions are given in section 6 .

\section{Brief overview of Hurricane Wilma}

Hurricane Wilma (2005), which formed over the Caribbean Sea, was the most intense Atlantic hurricane and the second-most intense TC recorded in the Western

\footnotetext{
${ }^{3}$ The animation can be accessed online (http://andrew.rsmas. miami.edu/bmcnoldy/tropics/earl10/Earl_30-31Aug10_SanJuan.gif).

${ }^{4}$ The animation can be accessed online (http://andrew.rsmas. miami.edu/bmcnoldy/tropics/maria17/Maria_19-20Sep17_TJUA.gif).
}

Hemisphere [after Hurricane Patricia (2015; Kimberlain et al. 2016)]. Its origin could be traced back to an unusually broad monsoon-like lower-tropospheric trough over much of the Caribbean Sea as early as 0000 UTC 11 October (Pasch et al. 2006; Chen et al. 2011). This trough was then split into two parts, with the southern one developing into a tropical depression (TD) near Jamaica by 1800 UTC 15 October when the surface circulation was well defined and the deep convection was sufficiently organized. In the subsequent few days, this TD was embedded in a region between a subtropical high over Mexico and the Gulf of Mexico, and another subtropical high over the North Atlantic Ocean. This synoptic configuration produced a weak steering flow, which drove the TD westward to west-southwestward for 1 day and then south-southwestward to southward for another 2 days. At 0600 UTC 17 October, the TD was upgraded to a tropical storm (TS) and named Wilma.

By early 18 October, Wilma's strengthening intensity reached hurricane level as it turned west-northwestward. Starting from 1800 UTC on the same day, Wilma underwent a remarkably rapid intensification (RI) process for a 12-h period until 0600 UTC 19 October. During this RI period, Wilma's central sea level pressure (SLP) dropped by $29 \mathrm{hPa}$ in the first $6 \mathrm{~h}$ and by $54 \mathrm{hPa}$ in the second $6 \mathrm{~h}$ (Fig. 1b). The minimum central SLP and the 1-min maximum sustained surface winds were $892 \mathrm{hPa}$ and $150 \mathrm{kt}\left(1 \mathrm{kt} \approx 0.51 \mathrm{~m} \mathrm{~s}^{-1}\right.$; category 5 on the SaffirSimpson hurricane wind scale), respectively, just after RI. During the RI stage, the U.S. Air Force reconnaissance observations indicated that Wilma's eye contracted to a diameter of $2 \mathrm{n} \mathrm{mi}(\sim 3.7 \mathrm{~km})$, which is the smallest eye known to the National Hurricane Center staff (Pasch et al. 2006). The intensification continued for another $6 \mathrm{~h}$. Wilma reached its peak intensity at 1200 UTC 19 October, with the 1-min maximum sustained surface winds of $160 \mathrm{kt}$, and the minimum central SLP of $882 \mathrm{hPa}$ (Fig. 1b) which broke the Atlantic record of $888 \mathrm{hPa}$ set by Hurricane Gilbert in 1988 (Lawrence and Gross 1989; Willoughby et al. 1989).

During the subsequent $24 \mathrm{~h}$, Wilma weakened from 882 to $910 \mathrm{hPa}$ because of an ERC. Then Wilma underwent another ERC before it made landfall on Cozumel Island (2145 UTC21 October) and then the northeastern Yucatan Peninsula. Consequently, Wilma weakened further to $960 \mathrm{hPa}$ over a 60 -h period from the completion of the first ERC, with 1-min maximum sustained surface winds of $85 \mathrm{kt}$ (0000 UTC 23 October). Around that time, Wilma turned north and arrived at the southern Gulf of Mexico. Subsequently, Wilma reintensified over the southeastern Gulf of Mexico when it turned to move northeastward, and then made a third landfall over southwestern Florida near Cape Romano around 1030 UTC 24 October with 1-min 

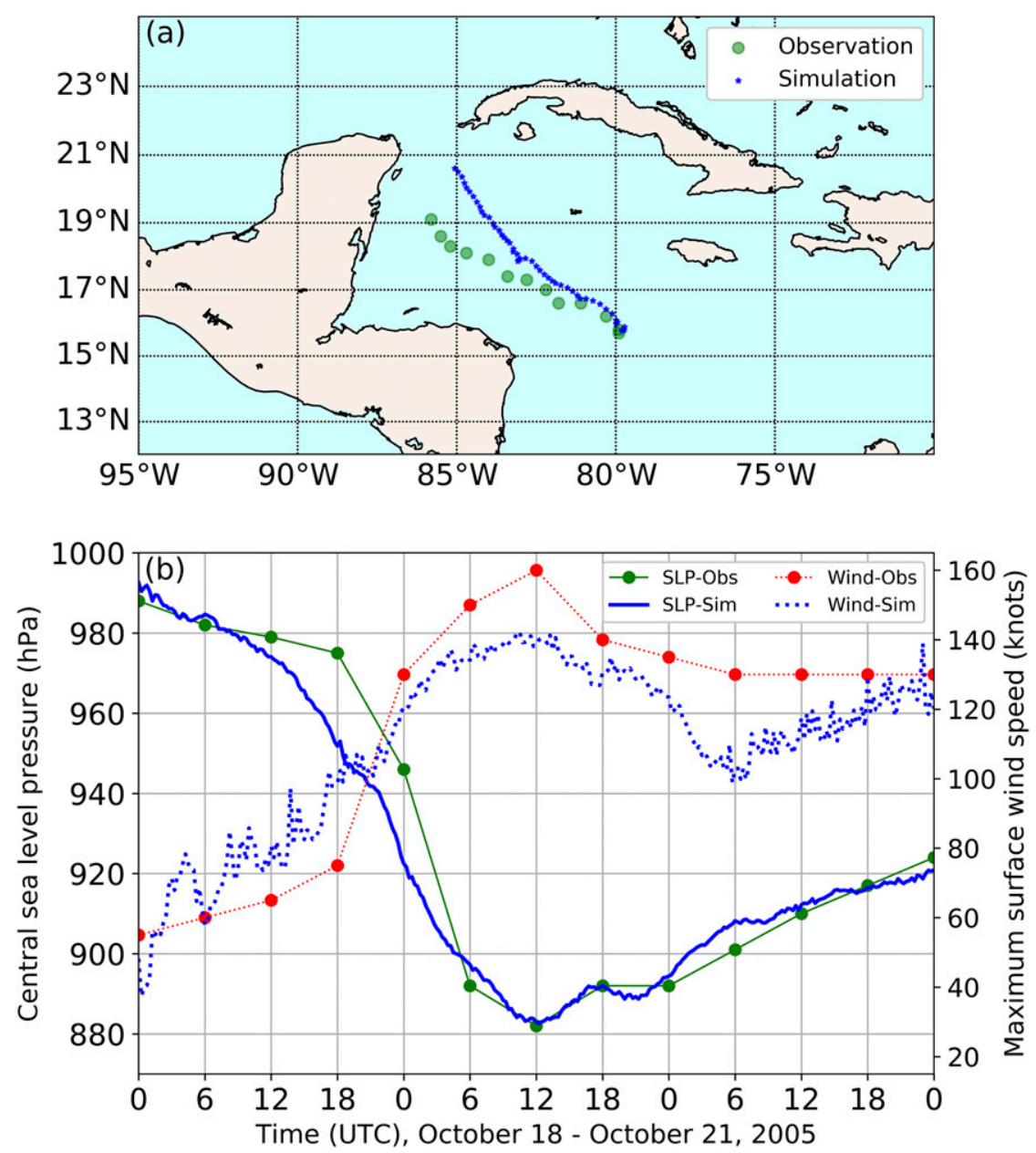

FIG. 1. Comparison between the WRF-simulated and observed Hurricane Wilma (2005) during the 72-h period of 0000 UTC 18 Oct-0000 UTC 21 Oct 2005 . The observation refers to the NHC best track data. (a) The 6-h observation (green circles) and the simulated track (blue stars). (b) Time series of observed TC central sea level pressure (green circles) compared with the simulation values (blue solid curve), and time series of observed TC maximum surface wind speed (red circles) compared with the simulation values (blue dotted curve). The plotting frequency for the simulation output is $30 \mathrm{~min}$.

maximum sustained surface winds of $105 \mathrm{kt}$. For more details, interested readers are referred to Pasch et al. (2006) and Chen et al. (2011).

\section{The numerical model and the simulation}

The simulation of Hurricane Wilma is performed using the Weather Research and Forecasting (WRF) Model, version 3.8.1 (Skamarock et al. 2008). WRF is a state-of-the-art three-dimensional full-physics compressible primitive equation system that has been widely used in the literature. The model is configured on a quadruply nested grid with two-way interaction. Only the innermost domain follows the TC vortex. The outermost domain consists of $140 \times 140$ horizontal grid points with a grid spacing of $27 \mathrm{~km}$. The nesting ratio is $3: 1$, such that the innermost domain has a grid spacing of $1 \mathrm{~km}$. For the 9-, 3-, and 1-km domains, the corresponding numbers of grid points are $286 \times 286,484 \times 484$, and $346 \times 346$, respectively. In the vertical direction, $30 \sigma$ levels are configured. The major model physics include the Thompson scheme for microphysics (Thompson et al. 2008), the Betts-Miller-Janjić cumulus parameterization scheme for deep convection (in the two outer domains only; Janjić 1994, 2000), the Mellor-Yamada-Janjić (Eta) TKE scheme for the planetary boundary layer (Janjić 1990, 1996, 2002), the RRTM scheme for longwave radiation (Mlawer et al. 1997), and the GSFC scheme for shortwave radiation (Chou and Suarez 1994). The initial and boundary conditions are supplied by the Geophysical 

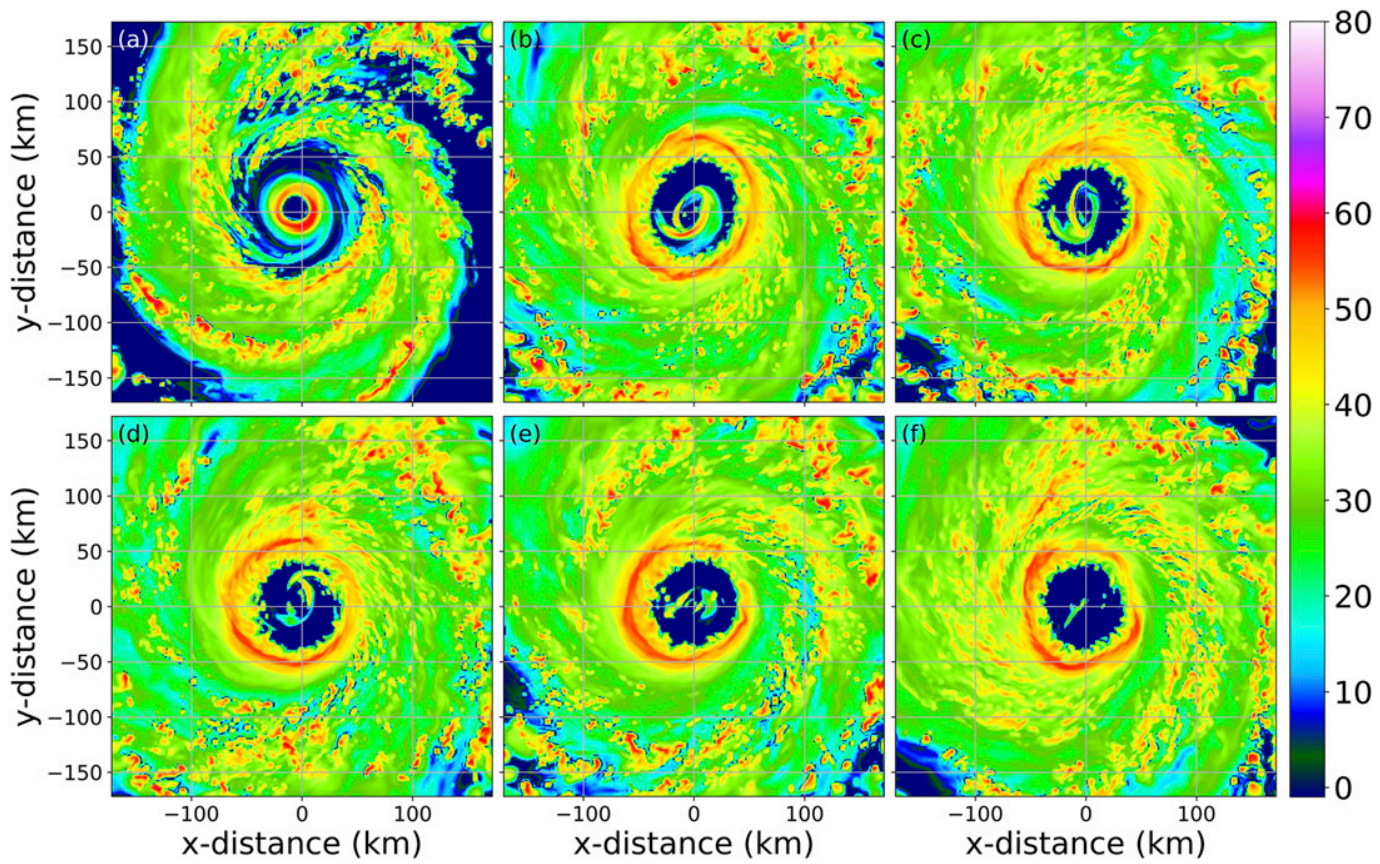

FIG. 2. The radar reflectivity (dBZ) of the simulated Wilma at $z=2 \mathrm{~km}$ at $t=$ (a) 42, (b) 59.5, (c) 62, (d) 64, (e) 66, and (f) $69 \mathrm{~h}$. The distance is with respect to the center of the innermost simulation domain.

Fluid Dynamics Laboratory (GFDL) hurricane model forecast data, and the surface conditions by the National Centers for Environmental Prediction (NCEP) Global Forecast System (GFS) final (FNL) analysis data. The constant sea surface temperature (SST) is given by the NCEP real-time global SST data with $0.5^{\circ}$ resolution at 0000 UTC 18 October 2005. The simulation is initialized at 0000 UTC 18 October 2005, and runs for $72 \mathrm{~h}$. It is worth mentioning that Wilma has been previously simulated by Chen et al. (2011), Menelaou et al. (2012), and Gadoury (2012).

\section{Simulation results}

Figure 1 displays the model-predicted storm track and intensity of Wilma together with the 6-hourly best track and the observed intensity issued by the National Hurricane Center (NHC; Pasch et al. 2006). It is clear that there is a northeastward bias in the simulated track in the late stages (approximately $185 \mathrm{~km}$ too far to the north-northeast of the best track at the end of the simulation; see Fig. 1a). However, the model reproduces the observed northwestward movement quite well. Note that this kind of track bias was also present in the simulations of Chen et al. (2011), Menelaou et al. (2012), and Gadoury (2012). Worthy of note is that the predicted and the observed evolution of central SLP show very good agreement, both in the trend and in the peak values (Fig. 1b). The discrepancy in maximum wind speed between the observation and simulation is larger, but the predicted maximum wind speed still captures the time of peak intensity. More specifically, the initial spinup, RI, and subsequent weakening stages are all captured by the model. Although the simulated RI started about $6 \mathrm{~h}$ earlier than the observation, and with a less intense deepening rate, the predicted peak intensity and time of occurrence are consistent with the best track.

Perhaps the most significant aspect of this simulation is that the model successfully captures the first SEF and the subsequent ERC, leading to the weakening of Wilma (see Fig. 2 for the radar reflectivity evolution at $z=2 \mathrm{~km}$ ). The simulated ERC lasted about $27 \mathrm{~h}$ (from 1800 UTC 19 October to 2100 UTC 20 October).

The outer eyewall of the actual Wilma (inferred from satellite microwave imagery $)^{5}$ formed around 1200 UTC 19 October and the inner eyewall dissipated around 1100 UTC 20 October. ${ }^{6}$ Note that during this time period,

\footnotetext{
${ }^{5}$ This satellite microwave imagery can be accessed online (https:// tropic.ssec.wisc.edu/real-time/mimic-tc/2005_TWENTY-FOUR/ webManager/mainpage.html).

${ }^{6}$ On the other hand, according to the weather reconnaissance aircraft vortex data messages, the observed first ERC of Wilma began at a time between 1822 and 2017 UTC 19 Oct, and ended at a time between 1046 and 1910 UTC 20 Oct. These vortex data messages can be obtained online (http://hurricanes.ral.ucar.edu/structure/vortex/ vdm_data/2005/vortex_AL252005_WILMA.txt).
} 

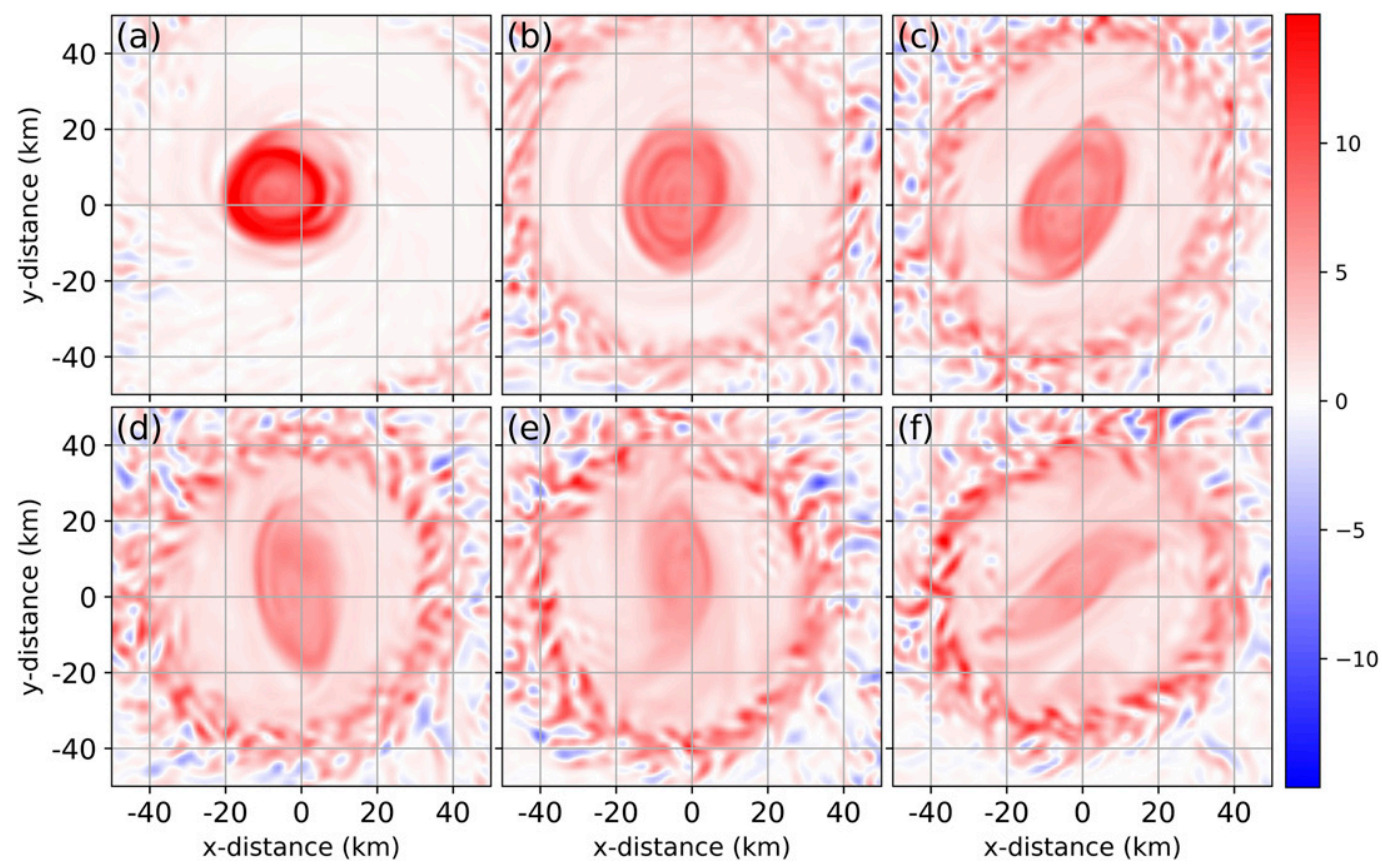

FIG. 3. The relative vorticity $\left(\times 10^{-3} \mathrm{~s}^{-1}\right)$ of the simulated Wilma averaged over the lower levels $0.8 \leq z \leq 2.5 \mathrm{~km}$ at $t=$ (a) 42 , (b) 54, (c) 59.5, (d) 62, (e) 64, and (f) $66 \mathrm{~h}$. The distance is with respect to the center of the innermost simulation domain. Note that the times and the scale of the axes are different from those in Fig. 2.

no land-based or airborne radar observations were available. Thus, relying solely on the relatively lowresolution satellite microwave imagery, we were unable to determine the degree of similarity between the simulated ERC and that of the real Wilma.

To set the stage for our analyses, it is appropriate to first examine the evolution of Wilma's secondary eyewall after it has been established. It should be noted that an eyewall is associated not only with the radar reflectivity maximum, but also the relative vorticity $\zeta$ maximum. As such, the evolution of both fields at three levels will be examined: the "lower levels," "middle levels," and "upper levels" refer respectively to the ranges from $z=800 \mathrm{~m}$ to $2.5 \mathrm{~km}$, from $z=2.5$ to $7.5 \mathrm{~km}$, and from $z=7.5$ to $11 \mathrm{~km}$. These ranges are determined subjectively from the evolution patterns of $\zeta$, with layers having analogous patterns categorized into the same group (not shown). ${ }^{7}$ Within the lower levels, the evolution and orientation of the elliptic inner eyewall is very similar. Moving up into the middle levels, this elliptical pattern gradually (but steadily) weakens and disappears. From the bottom layer of the upper levels, the trochoidal oscillation of the inner eyewall

\footnotetext{
${ }^{7}$ The corresponding center of each group is determined by the potential vorticity (PV) centroid (e.g., Ryglicki and Hart 2015).
}

becomes much more obvious. Note that at this stage, the analyses exclude any contribution from the boundary layer $[z<800 \mathrm{~m}$; in terms of the inflow layer depth as defined in Zhang et al. (2011)]. That being said, the extent to which the outcome of our current examination remains significant (or is modified) in the presence of a boundary layer requires further investigation. This additional investigation will be explored and reported in a separate paper.

Figure 3 shows the vorticity field averaged over the lower levels at six times starting from the time of SEF $(t \approx 42.0 \mathrm{~h}$; Fig. 3a). This SEF time is subjectively determined as the start time from which a complete outer ring of radar reflectivity emerges along with a clear and complete secondary ring of vorticity. It can be seen that after SEF, the outer eyewall begins to contract gradually (see also Fig. 2). Of interest is that by $t=59.5 \mathrm{~h}$ (Fig. 3c), the inner eyewall has deformed into a distinct elliptical structure. Subsequently, the deformation continues to amplify, resulting in an inner eyewall with a pronounced elliptical vorticity distribution. Eventually, the elongated inner eyewall vorticity patch touches the outer eyewall vorticity ring from $t \approx 63.5 \mathrm{~h}$ (e.g., Fig. 3e). Note that, during this period of elliptical elongation, the magnitude of the inner eyewall vorticity decreases (i.e., the color becomes paler) and the size of the inner eyewall vorticity patch largely decreases. (Figure 13, 

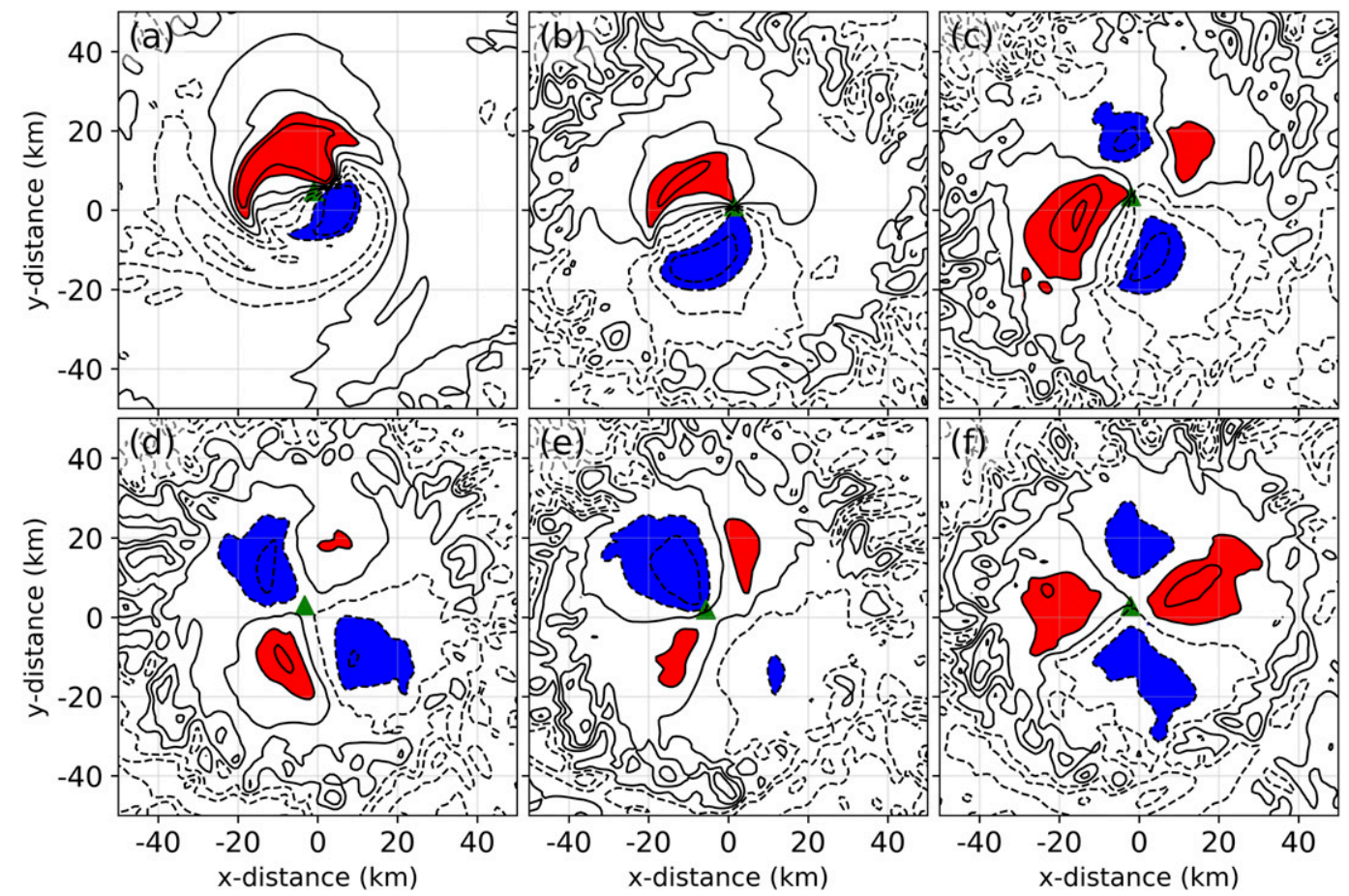

FIG. 4. The low-level radial velocity $\left(\mathrm{m} \mathrm{s}^{-1}\right)$ of the simulated Wilma averaged over the lower levels $(0.8 \leq z \leq 2.5 \mathrm{~km})$ at $t=$ (a) 42, (b) 59.0, (c) 59.5, (d) 62, (e) 64, and (f) $66 \mathrm{~h}$. Positive values are contoured from $2.5 \mathrm{~m} \mathrm{~s}^{-1}$ by $5 \mathrm{~m} \mathrm{~s}^{-1}$ (solid) while negative values are contoured from $-2.5 \mathrm{~m} \mathrm{~s}^{-1}$ by $-5 \mathrm{~m} \mathrm{~s}^{-1}$ (dashed). Green triangles mark the low-level center of the simulated Wilma. The red and blue shading highlight the radial outflow and radial inflow, respectively.

which will be discussed in section 5 , gives a clearer picture of the vorticity evolution after SEF.) Another salient feature is the oscillating wobbles exhibited by the inner eyewall. This is the primary reason as to why the location of the eye appears to deviate significantly from the geometric center of the outer eyewall [Figs. 2 and 3; readers are referred to Menelaou et al. (2018) for a discussion of a possible mechanism for oscillating wobbles].

\section{Evolution of radial flow and a possible dilution effect}

As mentioned, the period of elliptical deformation coincides with a simultaneous reduction of the inner eyewall vorticity. Based on this, it is reasonable to hypothesize a possible connection between the decay of the inner eyewall and this dominant asymmetry. To elaborate on the process of inner eyewall decay, the evolution of the radial flow at lower levels (based on Earth-relative winds) is shown in Fig. 4. After SEF, the radial velocity exhibits an azimuthal wavenumber-1 pattern. With time, and as the moat width decreases to a certain extent, the dominant pattern of the radial flow switches to a wavenumber-2 structure (at $t=$ 59.5 h; Fig. 4c). This wavenumber- 2 mode appears to be important as it represents the normal strain flow pattern which could lead to the dilution of the vorticity within the eye and the inner eyewall (the inner vorticity patch): there are a pair of radial outflow regions and a pair of radial inflow regions (e.g., Fig. 4d). By looking at Figs. 3 and 4 together, it is clear that the radial outflow pair acts to elongate the inner vorticity patch thereby inserting high- $\zeta$ air of the elongated part to the moat. Given that the elliptically elongated inner vorticity patch has significant cyclonic rotating motion relative to the moat, the $\zeta$ at the two ends of the inner vorticity patch can filament rapidly into small radial scales as they become axisymmetrized. The reason is that a moat is generally a region of active potential enstrophy cascade to small scales because of the presence of severe differential rotation (KSM00, and references therein). Meanwhile, the radial inflow pair acts to advect the low- $\zeta$ air from the moat into the region of the eye and the inner eyewall. This inward advection, if not short lived, can also greatly dilute the inner vorticity patch by mixing with the low- $\zeta$ air from the moat. Since the dominant wavenumber-2 pattern is retained for a long time, the pairs of radial inflow and outflow result in substantial dilution of the inner vorticity patch 
(i.e., the outer part of the inner vorticity patch largely mixes with the moat air, and therefore the area of inner vorticity patch decreases significantly). (For a more direct illustration of this dilution mechanism, readers are referred to section $5 \mathrm{c}$ and the schematic diagram in Fig. 20.) The discussion of the dynamics behind the wavenumber- 2 pattern is presented in the next section.

\section{Analyses and discussion}

\section{a. Linear stability analysis}

To study the barotropic aspect of the dynamics of a double-eyewall TC, a stability analysis of the linearized 2D nondivergent barotropic vorticity equation is performed. This equation, in polar coordinates $(r, \lambda)$, reads

$$
\left(\frac{\partial}{\partial t}+\bar{\omega} \frac{\partial}{\partial \lambda}\right) \nabla^{2} \psi^{\prime}-\left(\frac{1}{r} \frac{\partial \psi^{\prime}}{\partial \lambda}\right)\left(\frac{d \bar{\zeta}}{d r}\right)=0
$$

where $\bar{\zeta}(r)=(1 / r)\left(d\left(r^{2} \bar{\omega}\right) / d r\right)$ is the basic-state relative vorticity, $\bar{\omega}(r)=v(r) / r$ is the axisymmetric basic-state angular velocity, $\left(u^{\prime}, v^{\prime}\right)=\left[-(1 / r)\left(\partial \psi^{\prime} / \partial \lambda\right),\left(\partial \psi^{\prime} / \partial r\right)\right]$ are the perturbation radial and tangential components of velocity, $\nabla^{2} \psi^{\prime}$ is the perturbation vorticity $\zeta^{\prime}$, and $\psi^{\prime}$ is the perturbation streamfunction. After substituting the modal solutions of the form $\psi^{\prime}(r, \lambda, t)=\hat{\psi}(r) e^{i(m \lambda-\nu t)}$ into (1), where $m$ is the azimuthal wavenumber and $\nu$ is the complex frequency, the equation for the radial structure is obtained as follows:

$$
(\nu-m \bar{\omega})\left[r \frac{d}{d r}\left(r \frac{d \hat{\psi}}{d r}\right)-m^{2} \hat{\psi}\right]+m r \frac{d \bar{\zeta}}{d r} \hat{\psi}=0
$$

As previously mentioned, KSM00 performed a linear stability analysis for a TC-like vortex with a doubleeyewall structure. For their purpose, the system was idealized as a simple piecewise constant four-region model with a monopolar inner eyewall (region 1), the moat region (region 2), an annular secondary eyewall (region 3), and a far-field region with zero vorticity (region 4). We extended KSM00's model into a fiveregion model by replacing the monopolar inner eyewall

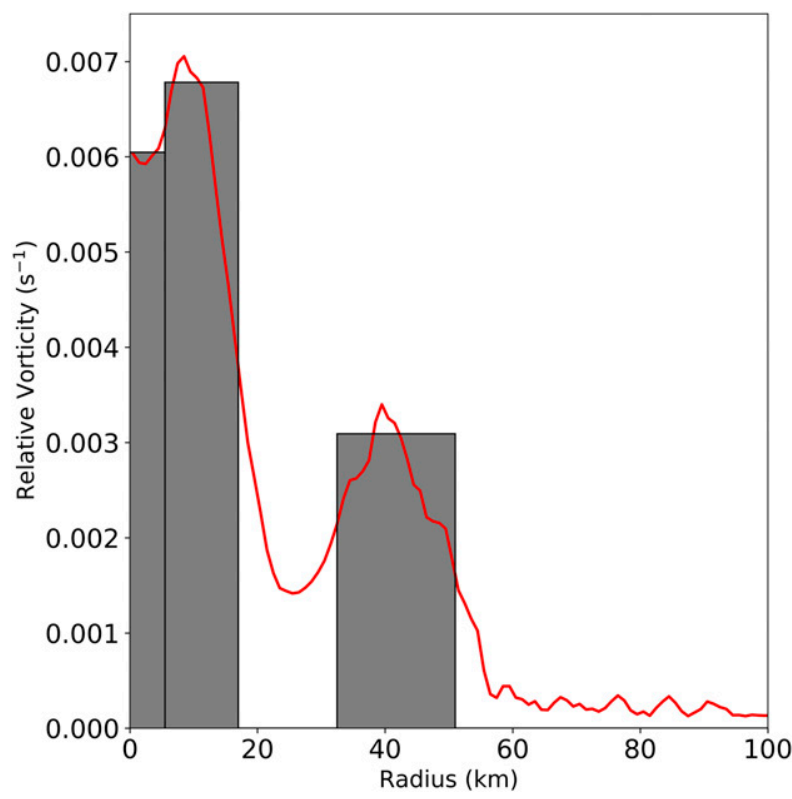

FIG. 5. The profile used for the linear stability analysis. The red curve represents the axisymmetric profile of simulated Wilma's relative vorticity at $\mathrm{t}=59.0 \mathrm{~h}$, which is approximated and simplified by the corresponding piecewise constant function (gray shading). The values used in this five-region approximation are $r_{0}=5.5$, $r_{1}=17, r_{2}=32.5$, and $r_{3}=51 \mathrm{~km}$.

by an annular inner eyewall similar to the simulated Wilma (see Figs. 2 and 3). Since a necessary condition for barotropic instability (Rayleigh's inflection point criterion) is that the basic-state vorticity gradient changes sign somewhere in the domain, it is clear that barotropic instability can be potentially excited in three regions throughout the vortex: one across the inner ring, one across the moat, and one across the outer ring.

To determine the parameters describing the fiveregion model, we display in Fig. 5 and Table 1 a comparison of the axisymmetric vorticity field of the simulated Wilma at $t=59.0 \mathrm{~h}$ ( $30 \mathrm{~min}$ prior to the change of the dominant wavenumber of low-level radial flow) and the step functions mimicking the structure in the five-region model used in the stability analysis. The angular velocity $\bar{\omega}(r)$ and the corresponding vorticity $\bar{\zeta}(r)$ in the five regions are given respectively by

$$
\bar{\omega}(r)=\frac{1}{2}\left\{\begin{array}{lc}
\zeta_{0}, & 0 \leq r \leq r_{0} \\
\zeta_{1}-\left(\zeta_{1}-\zeta_{0}\right)\left(r_{0} / r\right)^{2}, & r_{0} \leq r \leq r_{1} \\
\zeta_{2}-\left(\zeta_{2}-\zeta_{1}\right)\left(r_{1} / r\right)^{2}-\left(\zeta_{1}-\zeta_{0}\right)\left(r_{0} / r\right)^{2}, & r_{1} \leq r \leq r_{2} \\
\zeta_{3}-\left(\zeta_{3}-\zeta_{2}\right)\left(r_{2} / r\right)^{2}-\left(\zeta_{2}-\zeta_{1}\right)\left(r_{1} / r\right)^{2}-\left(\zeta_{1}-\zeta_{0}\right)\left(r_{0} / r\right)^{2}, & r_{2} \leq r \leq r_{3} \\
\zeta_{3}\left(r_{3} / r\right)^{2}-\left(\zeta_{3}-\zeta_{2}\right)\left(r_{2} / r\right)^{2}-\left(\zeta_{2}-\zeta_{1}\right)\left(r_{1} / r\right)^{2}-\left(\zeta_{1}-\zeta_{0}\right)\left(r_{0} / r\right)^{2}, & r \geq r_{3},
\end{array}\right.
$$


TABLE 1. The dimensionless vortex parameters of the simulated Wilma at $t=59.0 \mathrm{~h}$

\begin{tabular}{cl}
\hline \hline Parameters & Values \\
\hline$\frac{\zeta_{0}}{\zeta_{1}}$ & 0.88 \\
$\frac{\zeta_{1}}{\zeta_{3}}$ & 2.19 \\
$\frac{r_{0}}{r_{1}}$ & 0.3235 \\
$\frac{r_{1}}{r_{2}}$ & 0.5231 \\
$\frac{r_{2}}{r_{3}}$ & 0.6373 \\
\hline
\end{tabular}

and

$$
\bar{\zeta}(r)=\left\{\begin{array}{cc}
\zeta_{0}, & 0<r<r_{0} \\
\zeta_{1}, & r_{0}<r<r_{1} \\
\zeta_{2}, & r_{1}<r<r_{2} \\
\zeta_{3}, & r_{2}<r<r_{3} \\
0, & r>r_{3},
\end{array}\right.
$$

where $r_{0}, r_{1}, r_{2}$, and $r_{3}$ are the specified radii and $\zeta_{0}, \zeta_{1}, \zeta_{2}$, and $\zeta_{3}$ are the specified vorticity.

For the stability analysis, the interest is in the perturbation vorticity resulting from the radial displacement of the basicstate vorticity. The perturbation vorticity is nonzero only at the locations at the edges of the piecewise constant vorticity regions such that (2) reduces to $[r(d / d r)][r(d \hat{\psi} / d r)]-$ $m^{2} \hat{\psi}=0$ for $r \neq r_{0}, r_{1}, r_{2}$, and $r_{3}$. Following KSM00, the general solution (which is valid for all the five regions) consists of the linear combination of the basis functions:

$$
B_{j}^{(m)}(r)= \begin{cases}\left(r / r_{j}\right)^{m}, & 0 \leq r \leq r_{j} \\ \left(r_{j} / r\right)^{m}, & r \geq r_{j},\end{cases}
$$

for $j=0,1,2,3$, and

$\hat{\psi}(r)=\Psi_{0} B_{0}^{(m)}(r)+\Psi_{1} B_{1}^{(m)}(r)+\Psi_{2} B_{2}^{(m)}(r)+\Psi_{3} B_{3}^{(m)}(r)$,

where $\Psi_{0}, \Psi_{1}, \Psi_{2}$, and $\Psi_{3}$ are complex constants. It can be deduced from (5) that $d B_{j}^{(m)} / d r$ is discontinuous at $r=r_{j}$, and thus from the expression of perturbation vorticity $\nabla^{2} \psi^{\prime}$, that the solution associated with the coefficient $\Psi_{j}$ has vorticity anomalies concentrated at $r=r_{j}$. For relating $\Psi_{0}, \Psi_{1}$, $\Psi_{2}$, and $\Psi_{3}$, (2) is integrated over the narrow radial regions centered at $r_{0}, r_{1}, r_{2}$, and $r_{3}$ to obtain the jump condition:

$$
\lim _{\varepsilon \rightarrow 0}\left\{\left[\nu-m \bar{\omega}\left(r_{j}\right)\right] r_{j}\left(\frac{d \hat{\psi}}{d r}\right)_{r_{j}-\varepsilon}^{r_{j}+\varepsilon}\right\}+\left(\zeta_{j+1}-\zeta_{j}\right) m \hat{\psi}\left(r_{j}\right)=0
$$

where $j=0,1,2$, and 3. By substituting the general solution into (7), and considering a special case assuming that $\zeta_{2}=0$ (i.e., the relative vorticity within the moat is zero), an eigenvalue problem is obtained:

$$
\mathbf{M}\left(\begin{array}{c}
\Psi_{0} \\
\Psi_{1} \\
\Psi_{2} \\
\Psi_{3}
\end{array}\right)=\nu\left(\begin{array}{l}
\Psi_{0} \\
\Psi_{1} \\
\Psi_{2} \\
\Psi_{3}
\end{array}\right)
$$

where $\mathbf{M}$ is the matrix operator. To nondimensionalize this problem, divide (8) by $\zeta_{3} / 2$ to yield

$$
\mathbf{M}^{*}\left(\begin{array}{l}
\Psi_{0} \\
\Psi_{1} \\
\Psi_{2} \\
\Psi_{3}
\end{array}\right)=\nu^{*}\left(\begin{array}{l}
\Psi_{0} \\
\Psi_{1} \\
\Psi_{2} \\
\Psi_{3}
\end{array}\right)
$$

where $\mathbf{M}^{*}$ and $\nu^{*}=2 \nu / \zeta_{3}$ are the matrix operator and the eigenvalue, respectively, for the dimensionless eigenvalue problem in (9). The explicit expression of $\mathbf{M}^{*}$ and the discussion about solving (9) are provided in the appendix.

The corresponding solution for the case described in Fig. 5 is demonstrated in Figs. 6 and 7 in terms of the maximum value of the dimensionless growth rate $\nu^{*}$ (note the different scale on the ordinate axis for Fig. 7 vs Fig. 6). Here, two distinct types of barotropic instability can be seen. Type 1 (the left group in the diagrams) refers to the barotropic instability that operates across the outer ring and occurs for corresponding azimuthal wavenumbers $m=3,4,5, \ldots$; type 2 (the right group in the diagrams) is the outcome from the mutual growth of vortex Rossby waves across the moat region. The corresponding azimuthal wavenumbers are $m=2,3,4, \ldots$ An interesting note is that the two types of instability overlap in the central part of the diagrams and type 2 is the faster-growing instability for both regimes. According to Table 1 and Figs. 6 and 7, the simulated Wilma is most unstable to the wavenumber $m=2$ type- 2 instability at $t=59.0 \mathrm{~h}$. Needless to say, the aforementioned result might become different at different times throughout the life cycle of Wilma. For instance, if $r_{1} / r_{2}$ decreased to 0.35 (i.e., if the moat width increased) while keeping other parameters unchanged it can be inferred from the linear stability analysis diagrams that the $m=5$ type- 1 instability would dominate (in other words, the order of dominance switches). This may be also true for a different inner eyewall intensity (i.e., for a different $\zeta_{0} / \zeta_{1}$ and $\left.\zeta_{1} / \zeta_{3}\right)$. Figures 8 and 9 show another set of linear stability analysis diagrams for a much higher ratio $\zeta_{1} / \zeta_{3}=5.8$ and a lower ratio $\zeta_{0} / \zeta_{1}=0.67$ representing a 


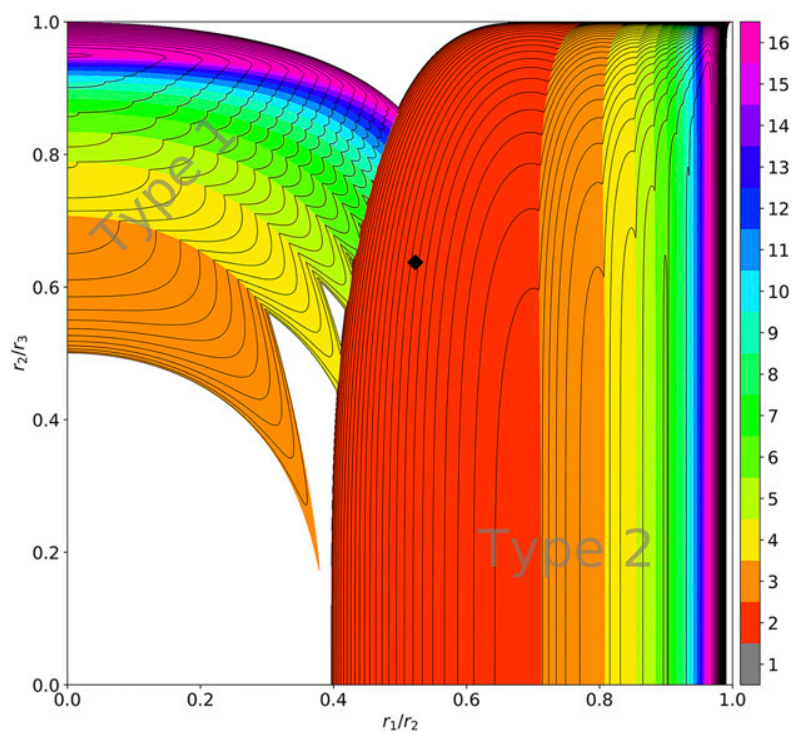

FIG. 6. Isolines of the maximum value of the dimensionless growth rate $\nu^{*}=2 \nu / \zeta_{3}$ computed from (A2), as a function of $r_{1} / r_{2}$ and $r_{2} / r_{3}$ for the case $\zeta_{1} / \zeta_{3}=2.3$ with $\zeta_{0} / \zeta_{1}=0.88$ and for azimuthal wavenumbers up to $m=16$. Type- 1 instability (for $m=3,4, \ldots, 16$ ) occurs on the left side of the diagram while type- 2 instability (for $m=2,3, \ldots, 16$ ) occurs on the right side. The color shading indicates the azimuthal wavenumber associated with the most unstable mode, with white color indicating the stable region. The minimum isoline value is $\nu^{*}=0.02$, and the contour interval is 0.02 . The black diamond indicates the radius ratios for the simulated Wilma's vorticity profile at $t=59.0 \mathrm{~h}$.

stronger inner eyewall. With every other vortex parameter remaining unchanged, the modified Wilma would be stable to any barotropic instabilities. Note that from Fig. 9, one can also identify a type-3 barotropic instability (which operates across the inner vorticity ring) - the one with azimuthal wavenumber $m$ that increases with $r_{0} / r_{1}$ (i.e., increases with decreasing thickness of the inner ring). For this hypothetical scenario with $\zeta_{0} / \zeta_{1}=0.67$ and $\zeta_{1} / \zeta_{3}=5.8$ (which are not valid for the simulated Wilma at $t=59.0 \mathrm{~h}$ ), the modified Wilma would be unstable to an $m=5$ type- 3 instability if $r_{0} / r_{1}$ was increased to about 0.44 (i.e., if the inner ring became thinner).

To summarize, at the time just before the low-level radial flow switches from an $m=1$ to an $m=2$ pattern, the simulated Wilma's vortex structure is most unstable to an $m=2$ type- 2 instability.

Before advancing, it is emphasized that the above analyses are linear and incorporate simplified step functions. In the following subsection, the dominance of type-2 instability in the simulated Wilma is further verified with nonlinear nondivergent barotropic vorticity experiments which consider continuous vorticity profiles.

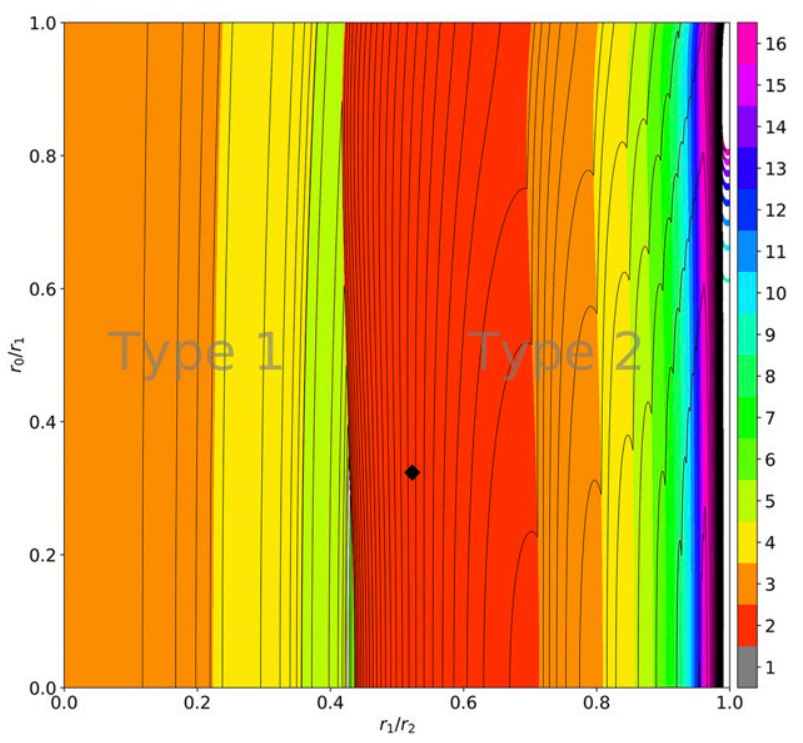

FIG. 7. Isolines of the maximum value of the dimensionless growth rate $\nu^{*}=2 \nu / \zeta_{3}$ computed from (A2), as a function of $r_{1} / r_{2}$ and $r_{0} / r_{1}$ for the case $\zeta_{1} / \zeta_{3}=2.3$ with $\zeta_{0} / \zeta_{1}=0.88$ and for azimuthal wavenumbers up to $m=16$. Type- 1 instability (for $m=3,4, \ldots, 16$ ) occurs on the left side of the diagram while type- 2 instability (for $m=2,3, \ldots, 16$ ) occurs on the right side. The color shading indicates the azimuthal wavenumber associated with the most unstable mode, with white color indicating the stable region. The minimum isoline value is $\nu^{*}=0.02$, and the contour interval is 0.02 . The black diamond indicates the radius ratios for the simulated Wilma's vorticity profile at $t=59.0 \mathrm{~h}$. Note that the ordinate in this figure is $r_{0} / r_{1}$ while that in Fig. 6 is $r_{2} / r_{3}$.

\section{b. Nonlinear nondivergent barotropic experiments}

\section{1) THE MODEL}

To determine whether a continuous axisymmetric vorticity field extracted from the Wilma simulation is susceptible to the $m=2$ type- 2 barotropic instability, a simple 2D unforced spectral nonlinear nondivergent barotropic vorticity (NDBV) model (Bartello and Warn 1996) is employed. The model is formulated in Cartesian coordinates and is based upon the prognostic equation for $\zeta$ on an $f$ plane. The governing equation is derived from

$$
\begin{aligned}
& \frac{\partial u}{\partial t}+u \frac{\partial u}{\partial x}+v \frac{\partial u}{\partial y}-f_{0} v=-\frac{\partial \phi}{\partial x}+\nu \nabla^{2} u \\
& \frac{\partial v}{\partial t}+u \frac{\partial v}{\partial x}+v \frac{\partial v}{\partial y}+f_{0} u=-\frac{\partial \phi}{\partial y}+\nu \nabla^{2} v \\
& \frac{\partial u}{\partial x}+\frac{\partial v}{\partial y}=0,
\end{aligned}
$$

where $(u, v)$ is the velocity in the $(x, y)$ direction, $\phi$ is the geopotential, $f_{0}$ is a constant Coriolis parameter, and $\nu$ is the kinematic viscosity. Using (10)-(12), the vorticity equation becomes 


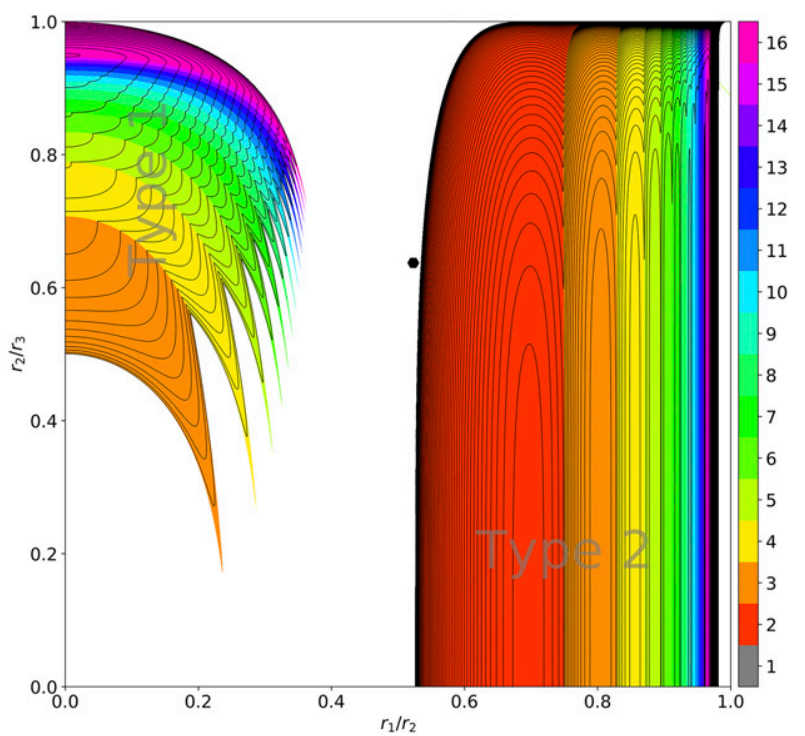

FIG. 8. Isolines of the maximum value of the dimensionless growth rate $\nu^{*}=2 \nu / \zeta_{3}$ computed from (A2), as a function of $r_{1} / r_{2}$ and $r_{2} / r_{3}$ for the hypothetical case $\zeta_{1} / \zeta_{3}=5.8$ with $\zeta_{0} / \zeta_{1}=0.67$ and for azimuthal wavenumbers up to $m=16$. Type-1 instability (for $m=3,4, \ldots, 16$ ) occurs on the left side of the diagram while type-2 instability (for $m=2,3, \ldots, 16$ ) occurs on the right side. The color shading indicates the azimuthal wavenumber associated with the most unstable mode, with white color indicating the stable region. The minimum isoline value is $\nu^{*}=0.02$, and the contour interval is 0.02 . The black hexagon indicates the radius ratios for the simulated Wilma's vorticity profile at $t=59.0 \mathrm{~h}$. Note that the vorticity ratios are different from those in Figs. 6 and 7.

$$
\frac{\partial \zeta}{\partial t}+\frac{\partial}{\partial x}(u \zeta)+\frac{\partial}{\partial y}(v \zeta)=\nu \nabla^{2} \zeta
$$

where $\zeta=\partial v / \partial x-\partial u / \partial y$. In the numerical modeling, the viscous term in (13) is replaced by the hyperviscous term $(-1)^{h-1} \nu_{h} \nabla^{2 h} \zeta$, where $\nu_{h}$ is the hyperviscosity coefficient and $h=2$ is the order of hyperviscosity. Equation (13) is solved with a Fourier pseudospectral code subject to doubly periodic boundary conditions. Time integration is performed with a standard leapfrog scheme. Note that the NDBV model does not impose a preference on any wavenumbers. In other words, the wavenumber- 2 mode is the fastest growing if the resultant evolution is in a wavenumber- 2 pattern.

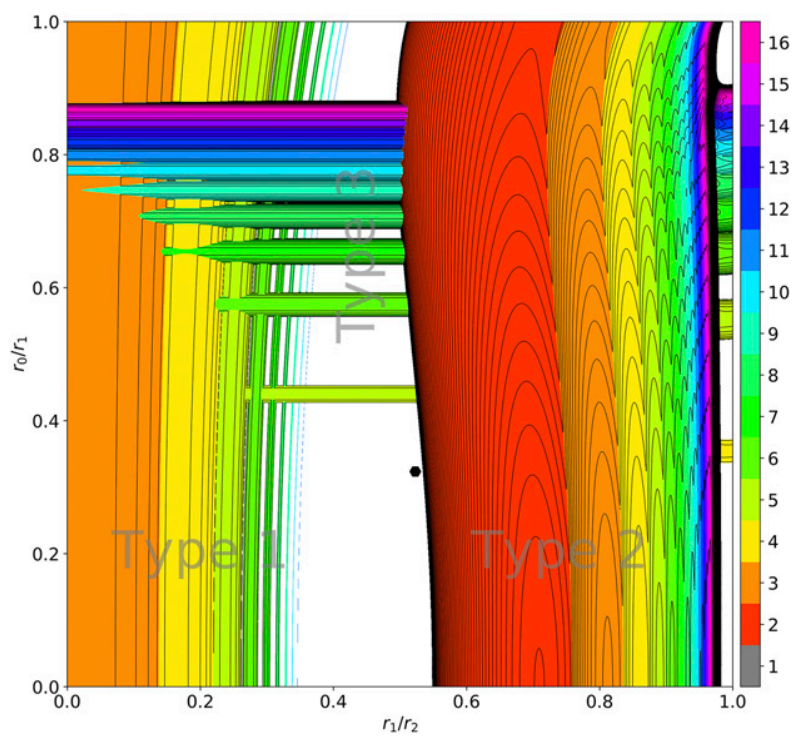

FIG. 9. Isolines of the maximum value of the dimensionless growth rate $\nu^{*}=2 \nu / \zeta_{3}$ computed from (A2), as a function of $r_{1} / r_{2}$ and $r_{0} / r_{1}$ for the hypothetical case $\zeta_{1} / \zeta_{3}=5.8$ with $\zeta_{0} / \zeta_{1}=0.67$ and for azimuthal wavenumbers up to $m=16$. Type-1 instability (for $m=3,4, \ldots, 16)$ occurs on the left side of the diagram while type-2 instability (for $m=2,3, \ldots, 16$ ) occurs on the right side. Type-3 instability occurs in the top part of the diagram, and it dominates over type- 1 but is dominated over by type-2. The color shading indicates the azimuthal wavenumber associated with the most unstable mode, with white color indicating the stable region. The minimum isoline value is $\nu^{*}=0.02$, and the contour interval is 0.02 . The black hexagon indicates the radius ratios for the simulated Wilma's vorticity profile at $t=59.0 \mathrm{~h}$.

\section{2) THE EXPERIMENTS}

To examine the dominant instability type (and characteristics) to which a particular vorticity field is susceptible, the following steps are adopted: At each desired time, the vorticity field from the 1-km WRF simulation domain $(346 \times 346$ grid points $)$ is extracted and extrapolated to a domain with $360 \times 360$ grid points. The resultant field is then azimuthally averaged, vertically averaged (over a range of selected heights), and fed into the spectral NDBV model. This initial axisymmetric vortex is perturbed by a small perturbation that is added across the moat region and has the following form:

$$
\zeta^{\prime}(r, \lambda)=\zeta_{\text {amp }} \sum_{m=1}^{12} \cos (m \lambda)\left\{\begin{array}{lc}
0, & 0 \leq r \leq r_{1}-d \\
S\left[\left(r_{1}+d-r\right) / 2 d\right], & r_{1}-d \leq r \leq r_{1}+d \\
1, & r_{1}+d \leq r \leq r_{2}-d \\
S\left[\left(r-r_{2}+d\right) / 2 d\right], & r_{2}-d \leq r \leq r_{2}+d \\
0, & r \geq r_{2}+d,
\end{array}\right.
$$

where $\zeta_{\text {amp }}$ is a specified constant equivalent to $0.5 \%$ of the minimum vorticity in the moat, $d=5 \mathrm{~km}$ is a specified quantity, and $S(s)=1-3 s^{2}+2 s^{3}$ is the basic cubic Hermite shape function satisfying $S(0)=1, S(1)=0$, 
and $S^{\prime}(0)=S^{\prime}(1)=0$. Here, the spectral model is specified to have $360 \times 360$ equally spaced collocation points on a $360 \mathrm{~km} \times 360 \mathrm{~km}$ domain. Each experiment is executed with a dealiased calculation of the quadratic terms in (13), which yields $120 \times 120$ Fourier modes. The wavelength of the highest Fourier mode, which is $3 \mathrm{~km}$, is a realistic estimate of resolution. The total integration time for each case is $18 \mathrm{~h}$.

Before proceeding, recall that after Wilma's secondary eyewall has been established at $t \approx 42.0 \mathrm{~h}$, the dominant low-level radial flow pattern switches from $m=1$ to $m=2$ at $t=59.5 \mathrm{~h} .{ }^{8}$ The transition implies that during the time just before the transition, Wilma's low-level $\zeta$ structure might have been subjected to the $m=2$ barotropic instability excited across the moat region. To verify whether this is a reasonable hypothesis, a group of two experiments is conducted using the NDBV model. In the first experiment (denoted by experiment A1), the model is initialized with Wilma's axisymmetric lowlevel vorticity profile (following the abovementioned process) $6 \mathrm{~h}$ before the $m=2$ transition (at $t=53.5 \mathrm{~h}$ ). On the other hand, in the second experiment (denoted by experiment A2) the model is initialized with Wilma's vorticity profile only $30 \mathrm{~min}$ before the transition. The initial axisymmetric vorticity profiles of all NDBV experiments are shown in Fig. 10.

Figure 11 depicts the results of experiment $\mathrm{A} 1$ in the form of total vorticity maps. From Fig. 11a, it can be seen that the $m=2$ perturbation across the moat becomes obvious at $t_{\mathrm{NDBV}}=9.0 \mathrm{~h}$. The inner core (inner vorticity patch) becomes slightly elliptic at $t_{\mathrm{NDBV}}=12.0 \mathrm{~h}$. As per Fig. 11b, there is only weakly exponential growth with growth rate $\nu=4.8 \times 10^{-5} \mathrm{~s}^{-1}$ (e-folding time of $5.8 \mathrm{~h}$ ). Figure $12 \mathrm{a}$ shows the total and perturbation vorticity of A2 over the same period. In contrast to A1, an $m=2$ pattern becomes clearly evident by $3 \mathrm{~h}$ (both at the inner core and the moat region) with significant ellipticity. Of importance is that the $\zeta^{\prime}$ pattern at this time (the top-right panel of Fig. 12a) indicates a phase difference of $90^{\circ}$ between the $m=2$ vortex Rossby waves (VRWs) at the outer edge of the inner eyewall and the inner edge of the outer eyewall. This is the signature of a type- 2 barotropic instability according to the theory of VRWs (e.g., Hoskins et al. 1985; Schubert et al. 1999). On the other hand, a type-3 instability does not emerge as indicated by the phase difference of $180^{\circ}$ between the $m=2 \mathrm{VRW}$ at the inner and outer edges of the inner eyewall. In addition, experiment A2 exhibits a rapid exponential growth of

\footnotetext{
${ }^{8}$ Radial flow pattern of $m=2$ associated with type-2 instability emerges and develops from $t=57.0 \mathrm{~h}$ (not shown). It becomes dominant at $t=59.5 \mathrm{~h}$.
}

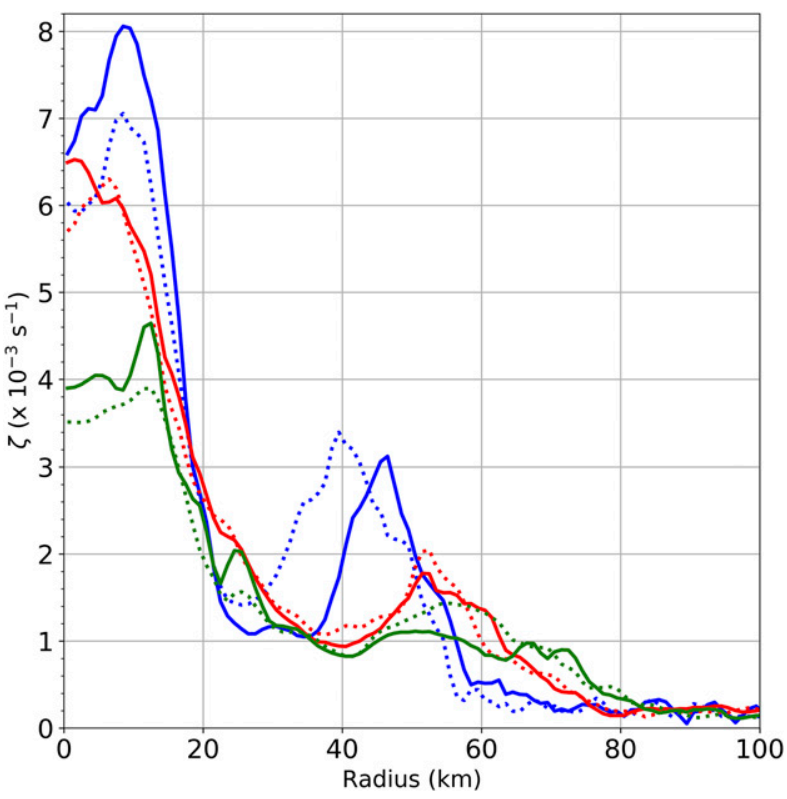

FIG. 10. The initial axisymmetric $\zeta$ profiles of all NDBV experiments: A1 (solid blue), A2 (dotted blue), B1 (solid red), B2 (dotted red), C1 (solid green), and C2 (dotted green).

the perturbation (Fig. 12b; $e$-folding time of $0.7 \mathrm{~h}$ ). By combining the results of $\mathrm{A} 1$ and $\mathrm{A} 2$, it is highly suggestive that the vorticity profile at $t=59.0 \mathrm{~h}$ is indeed highly susceptible to the type-2 barotropic instability (with $m=$ 2 being the fastest-growing asymmetry) whereas there is only weak instability at $t=53.5 \mathrm{~h}$. Note further that the main difference between the two initial vorticity profiles resides in the moat width (cf. the solid blue curve with the dotted blue curve in Fig. 10). At $t=53.5 \mathrm{~h}$, the moat width is larger relative to its counterpart at $t=59.0 \mathrm{~h}$ (experiment A1: approximately $23 \mathrm{~km}$; experiment A2: approximately $15 \mathrm{~km}$ ). This indicates that a type- 2 barotropic instability becomes vigorous when the moat width decreases (recall that the outer eyewall gradually contracts radially with time) to a value such that $r_{1} / r_{2}$ falls within a more unstable regime computed by the linear stability analysis (Figs. 6 and 7). This result is further supported by a linear stability analysis (not shown) for the $\zeta$ field at $t=53.5 \mathrm{~h}$ (the same profile for experiment A1), which consistently shows that the vorticity profile lies much closer to the stable region of the stability phase space than that at $t=59.0 \mathrm{~h}$. Overall, the NDBV experiments support the excitation of a type-2 barotropic instability in the simulated Wilma (2005) prior to (but near) $t=59.5 \mathrm{~h}$.

\section{c. Evolution of the strength of the inner vorticity patch}

Although we have established that the $m=2$ type-2 barotropic instability was indeed excited in 
(a)
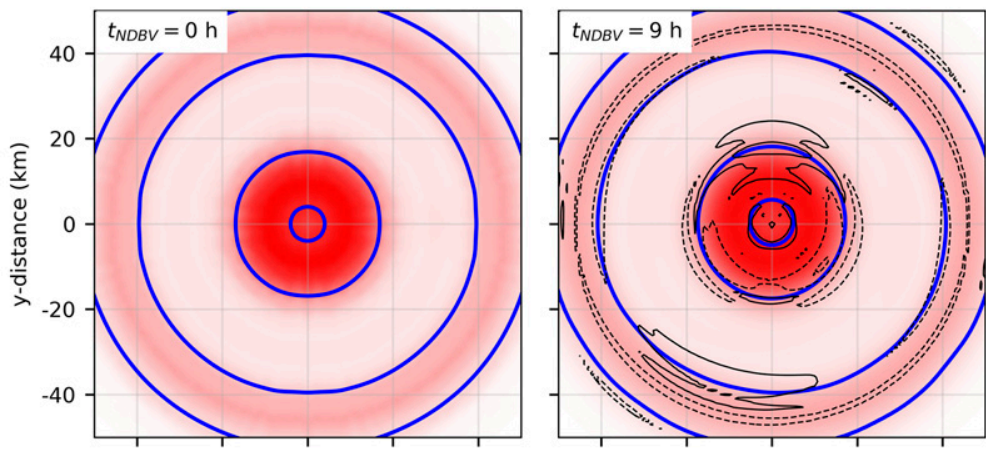

$-0.008$
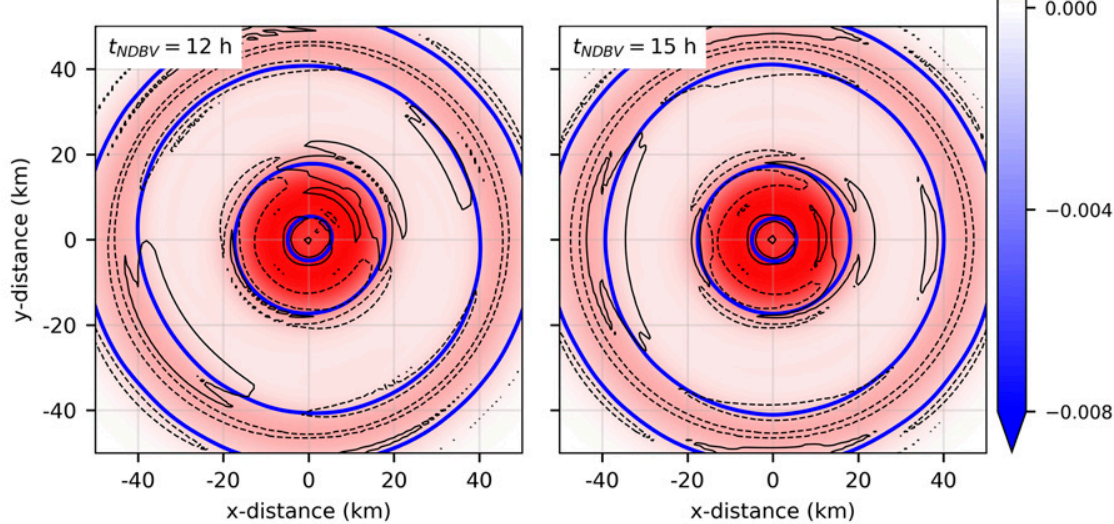

(b)

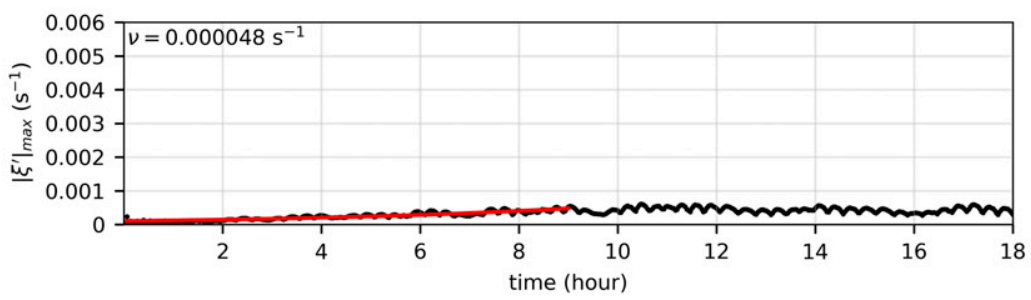

FIG. 11. NDBV experiment A1. (a) Relative vorticity $\zeta$ (shading) and its perturbation $\zeta^{\prime}$ (black contours) at the indicated experiment times. Positive values (solid) are contoured from $\zeta^{\prime}=1.5 \times 10^{-4} \mathrm{~s}^{-1}$ by $3 \times 10^{-4} \mathrm{~s}^{-1}$ while negative values (dashed) are contoured from $\zeta^{\prime}=-1.5 \times 10^{-4} \mathrm{~s}^{-1}$ by $-3 \times 10^{-4} \mathrm{~s}^{-1}$. The $m \geq 5$ patterns of the $\zeta^{\prime}$ field are filtered out, given that an azimuthal Fourier analysis shows $m<5$ patterns are dominant. The blue contours of $\partial^{2} \zeta / \partial r^{2}=0$ outline the shape of the outer eyewall, the moat, and the inner eyewall. The distance is with respect to the center of the vortex. (b) The time series of the maximum absolute values of the perturbation relative vorticity $\left|\zeta^{\prime}\right|_{\max }$ (black dots). The red curve is the exponential fit for the corresponding period and $\nu$ is the corresponding growth rate. The abscissa is the experiment time.

the simulated Hurricane Wilma, it remains to be determined whether its long-term nonlinear effect contributed to the weakening (and eventually the dissipation) of the inner vorticity patch (or the inner eyewall). To this end, we examine the time series (after SEF) of the circulation of the inner vorticity patch $C_{\mathrm{in}}=\iint_{A} \zeta d A$, where $A$ is the area of the inner vorticity patch.
The calculation of $C_{\text {in }}$ merits some careful consideration. As a prerequisite, the region over which $C_{\text {in }}$ is calculated must be defined in a quantitative manner. In this paper, it is designated to satisfy $r<r_{\text {th }}$ and $\zeta>\zeta_{\text {th }}$, where $r_{\text {th }}$ is a threshold radius such that the outer eyewall is excluded, and $\zeta_{\text {th }}$ is a threshold relative vorticity (defined at each level) such that only the vorticity patch associated with the inner eyewall is recognized. 
(a)
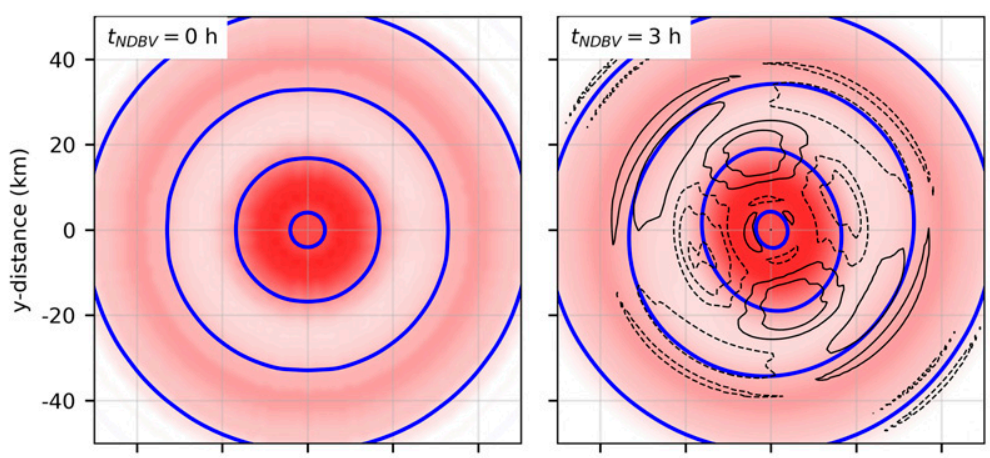

$-0.008$
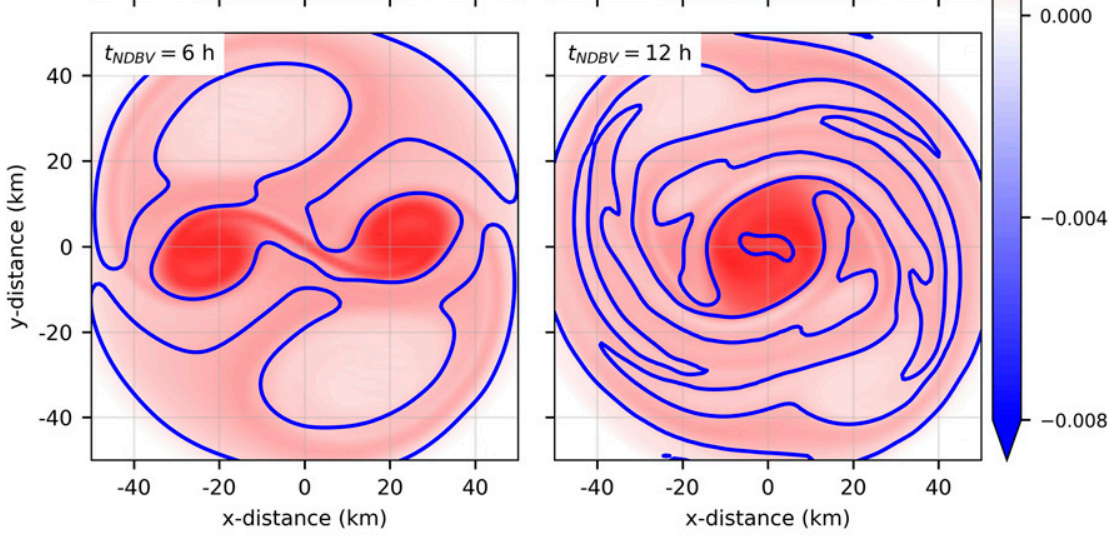

(b)

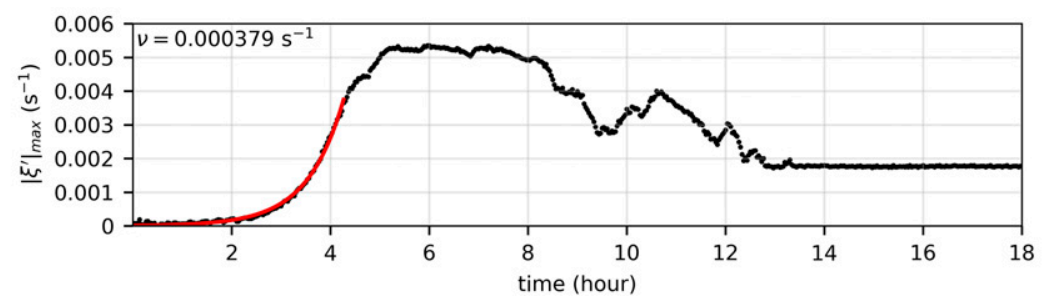

FIG. 12. As in Fig. 11, but for NDBV experiment A2. (a) Note that black contours are not plotted in the bottom panels since $\zeta^{\prime}$ becomes intricate as the nonlinear development continues.

According to the above relations, for the inner vorticity patch to be a reasonable surrogate for the time-evolving vorticity during the targeted decay phase of the inner eyewall, $\zeta_{\text {th }}$ is determined based on the magnitude of the inner vorticity patch just prior to the completion of the ERC (in other words, the last simulation output step having a discernible inner eyewall). Considering the fact that the inner eyewall vorticity follows an overall decline trend while the moat region vorticity follows an overall increase trend after the SEF (see section 4), the aforementioned threshold yields accurately the $C_{\text {in }}$ throughout the whole period of interest. Note that, $C_{\text {in }}$ intrinsically includes two pieces of information: the intensity and the size of the inner vorticity patch (both needed in order to determine the strength of the inner vorticity patch realistically). ${ }^{9}$

Figure 13 shows the time evolution of $C_{\text {in }}$ for the three groups of tropospheric layers (lower, middle, and upper). Shortly after SEF (around $t=45.0 \mathrm{~h}$ ), $C_{\text {in }}$ begins to

\footnotetext{
${ }^{9}$ One may also study the strength evolution of the inner vorticity patch in terms of its spatial mean $\langle\zeta\rangle$. However, it is a less appropriate choice for this study because it is shown in the simulation (see Fig. 3) that the outer part of the inner vorticity patch is largely diluted (such that $\zeta<\zeta_{\text {th }}$ there) while the inner part is much less affected. In this case, $\langle\zeta\rangle$ may not decrease accordingly because the largely diluted and/ or sheared part is no longer considered as a part of the inner vorticity patch. On the other hand, the effect of size change is included when using $C_{\text {in }}$. Therefore, $C_{\text {in }}$ is a better choice over $\langle\zeta\rangle$.
} 


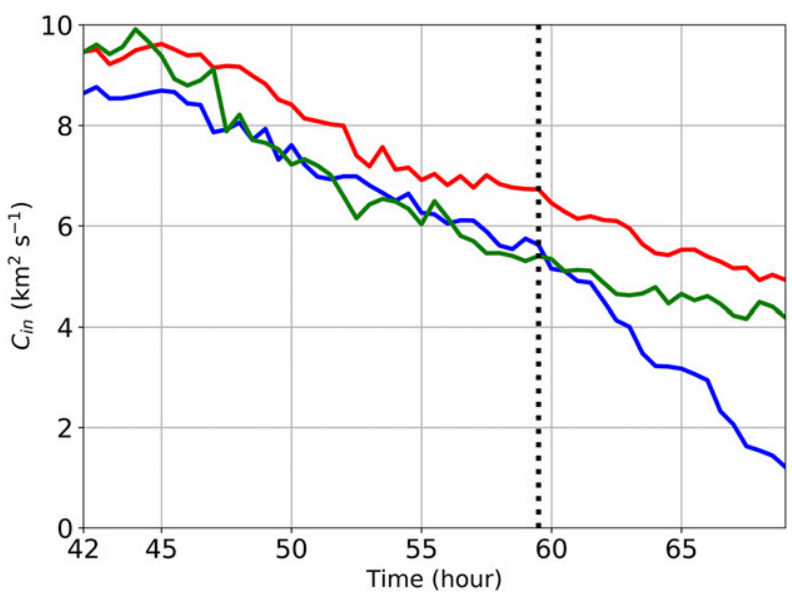

FIG. 13. Time series of the circulation $\left(\mathrm{km}^{2} \mathrm{~s}^{-1}\right)$ of the inner vorticity patch $C_{\text {in }}$ calculated from the WRF simulation output, averaged over the lower levels (blue), middle levels (red), and upper levels (green). The black dotted line indicates the start time $(t=59.5 \mathrm{~h})$ of the second period of significant decrease in low-level $C_{\text {in }}$. This line also marks the start of the long-lasting period in which the dominant low-level radial flow pattern is wavenumber 2 . The abscissa is the simulation time since 0000 UTC 18 Oct 2005.

decrease throughout the whole column. Such behavior over the entire troposphere may imply that the decay during this time is largely associated with the establishment of the outer eyewall. Nevertheless, nearly $14.5 \mathrm{~h}$ later, at around $t=59.5 \mathrm{~h}$ it can be seen that $C_{\text {in }}$ at the lower levels undergoes a second period of a much faster decrease. For instance, from $t=59.5$ to $67.5 \mathrm{~h}$ the decline rate of $C_{\text {in }}$ is about $0.503 \mathrm{~km}^{2} \mathrm{~s}^{-1} \mathrm{~h}^{-1}$, whereas from $t=45.0$ to $59.5 \mathrm{~h}$ the decline rate ${ }^{10}$ is about $0.216 \mathrm{~km}^{2} \mathrm{~s}^{-1} \mathrm{~h}^{-1}$. While the mean vorticity of inner vorticity patch $\langle\zeta\rangle$ keeps decreasing after $t=59.5 \mathrm{~h}$, the sharper decrease in the area of the inner vorticity patch contributes the most to this much faster decrease in $C_{\text {in }}$ (not shown). Of particular interest is that the initiation time (of this second period of significant decrease) coincides with the time when $m=2$ radial flow pattern associated with type-2 instability becomes dominant at the lower levels (see section 5b). This nearly exact match strongly suggests that this instability results in a considerable decay of the inner vorticity patch as manifested by the sharper decrease in $C_{\mathrm{in}}$. Based on

\footnotetext{
${ }^{10}$ The decline rate of $C_{\text {in }}$ in NDBV experiment $\mathrm{A} 2$ is $0.121 \mathrm{~km}^{2} \mathrm{~s}^{-1} \mathrm{~h}^{-1}$. NDBV experiment A2 is repeated as a preliminary WRF idealized experiment (with all model physics switched off) initialized with a barotropic vortex having the same axisymmetric profile as that in NDBV experiment A2. The resultant decline rate of $C_{\text {in }}$ during the fast-decay period is $0.389 \mathrm{~km}^{2} \mathrm{~s}^{-1} \mathrm{~h}^{-1}$. A thorough investigation will be reported in a future paper.
}

these characteristics, it is proposed that under the $m=2$ type- 2 instability the decay of inner vorticity patch results from the asymmetric radial inflow and outflow that effectively mix high $\zeta$ from the outer part of the inner vorticity patch with low $\zeta$ from the moat. This dilution (or mixing) process makes the inner vorticity patch shrink quickly. As an aside, there seems to be two transient periods (from $t=64.0$ to $65.5 \mathrm{~h}$ and from $t=67.5$ to $68.5 \mathrm{~h}$ ) in which the weakening of the low-level $C_{\text {in }}$ nearly ceases. It turns out that this feature is mainly caused by the radial flows during these times switching back to an $m=1$ pattern (figure not shown). ${ }^{11}$ The dynamics behind such behavior (transition back and forth between $m=1$ and $m=2$ ) is an intriguing feature which will be investigated in the future.

\section{d. Evolution of the inner vorticity patch at the middle and upper levels}

Returning to Fig. 13, it is evident that in contrast to the lower levels, the vorticity at the middle and upper levels did not experience a second period of faster weakening. To investigate this difference, we examine the radial flows at the middle and upper levels in Figs. 14 and 15 (the corresponding low-level field is shown in Fig. 4). At the middle levels at $t=59.5 \mathrm{~h}$ (Fig. 14a), the radial flow is characterized by an $m=2$ structure. However, this feature appears short lived as by $t=60.5 \mathrm{~h}$, the dominant wavenumber pattern switches to $m=1$ which remains the significant asymmetry until the end of the simulation. At the upper levels, the flow is characterized by an $m=1$ pattern all the time.

To explain why the $m=2$ pattern fails to be dominant at the middle and upper levels, two groups of additional experiments will be conducted with the NDBV model initialized with a number of vorticity profiles extracted from the mid- (group B experiments) and upper levels (group C experiments) of the WRF simulation. Each group consists of two independent experiments. Within group B, experiment B1 is initialized with an axisymmetric vorticity profile when the dominant radial flow structure transitions from $m=1$ to $m=2$ (at $t=59.0 \mathrm{~h})$. Experiment B2 is initialized with a vorticity profile right after the flow transitions back to $m=1$ (at $t=60.0 \mathrm{~h})$.

\footnotetext{
${ }^{11}$ The $m=1$ instability, which manifests itself as the trochoidal oscillation of the eye of a single-eyewall TC, is reported in Nolan and Montgomery (2000, and references therein), Nolan et al. (2001, and references therein), and Schecter and Montgomery (2007). As a contrast, the $m=1$ pattern in our WRF simulation is due to the $m=1$ instability between the inner and outer eyewalls leading to the trochoidal oscillation of the inner eyewall as a whole within the secondary eyewall (Oda et al. 2006; Menelaou et al. 2018).
} 


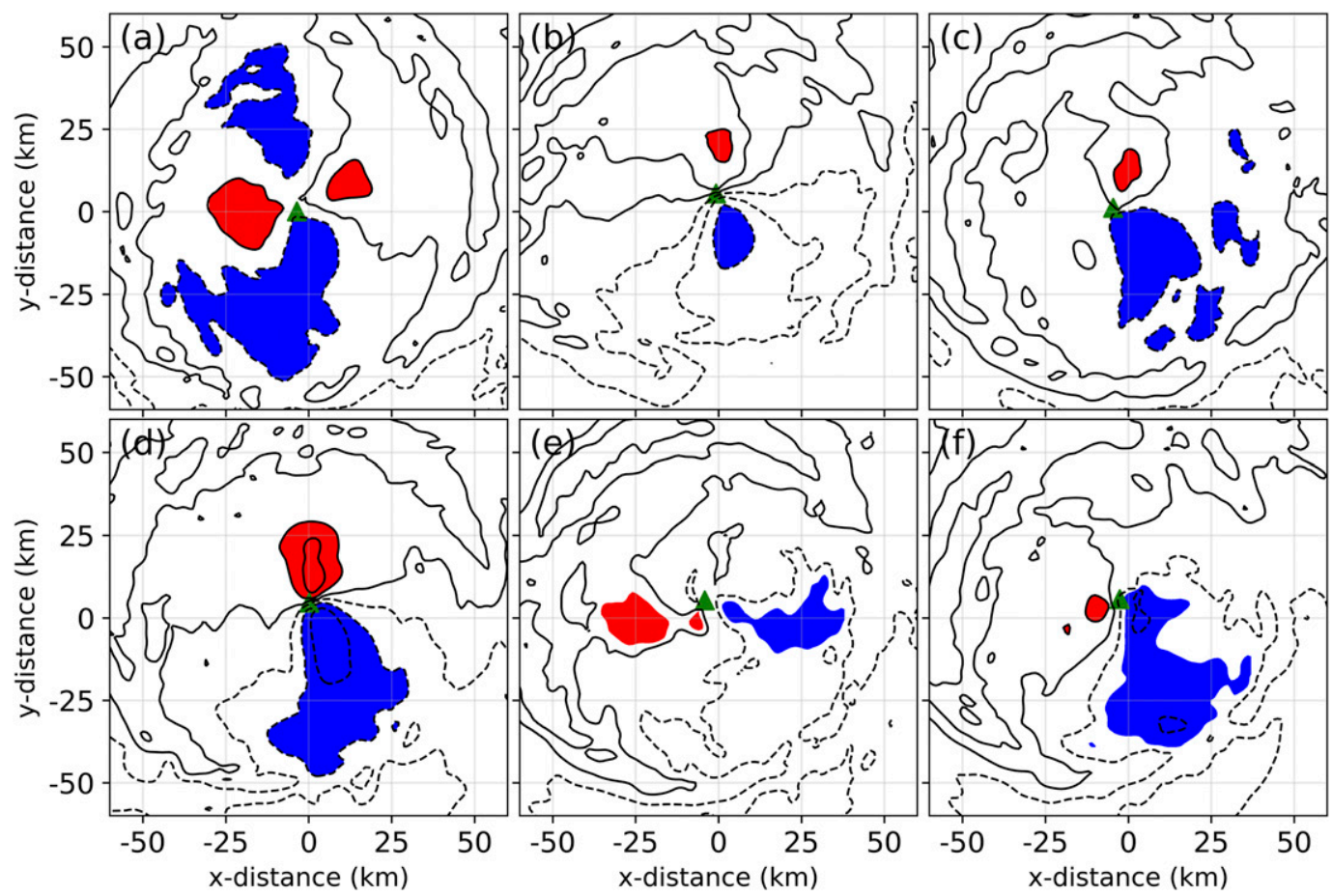

FIG. 14. The radial velocity $\left(\mathrm{m} \mathrm{s}^{-1}\right)$ of the simulated Wilma averaged over the middle levels $(2.5 \leq z \leq 7.5 \mathrm{~km})$ at $t=$ (a) 59.5, (b) 60.5, (c) 61.5, (d) 62, (e) 64, and (f) $66 \mathrm{~h}$. Note that the times are different from those in Fig. 4. Positive values are contoured from $2.5 \mathrm{~m} \mathrm{~s}^{-1}$ by $5 \mathrm{~m} \mathrm{~s}^{-1}$ (solid) while negative values are contoured from $-2.5 \mathrm{~m} \mathrm{~s}^{-1}$ by $-5 \mathrm{~m} \mathrm{~s}^{-1}$ (dashed). Green triangles mark the midlevel center of the simulated Wilma. The red and blue shading highlight the radial outflow and radial inflow respectively. The color shading in (e) and (f) is produced with a different set of contour levels. The distance is with respect to the center of the innermost simulation domain.

Figures 16a and 17a depict respectively the B1 and B2 total and perturbation vorticity at selected times. In B1, the vortex retains its axisymmetric structure for around $7 \mathrm{~h}$. By $t_{\mathrm{NDBV}}=9.0 \mathrm{~h}$, a clear $m=2$ asymmetry has been excited (Fig. 16a). This characteristic vortex deformation implies that the WRF vorticity profile at the middle levels is susceptible to a type-2 instability. Despite this, there is a large difference in the growth rate between $\mathrm{B} 1$ and $\mathrm{A} 2$ (in which an $m=2$ asymmetry becomes discernible by $t_{\mathrm{NDBV}}=3.0 \mathrm{~h}$ ). The estimated $e$-folding time from the fitted exponential curve for A2 (the red curve in Fig. 12b) is about $0.7 \mathrm{~h}$ while that for B1 (the red curve in Fig. 16b) is much longer-around $6.3 \mathrm{~h}$. This different growth rate indicates that the vortex at the middle levels is significantly more stable relative to its low-level counterpart. Additionally, the vortex in B2 becomes slightly asymmetric at $t_{\mathrm{NDBV}}=12.0 \mathrm{~h}$ with a slower growth rate (longer $e$-folding time of $9.0 \mathrm{~h}$ ). Taken together, the group B experiments indicate that the midlevel vorticity profile of the simulated Wilma is marginally stable and hence a dominant $m=2$ radial flow pattern cannot be sustained.
Regarding group C (figure not shown), experiment $\mathrm{C} 1$ is initialized with an upper-level vorticity profile extracted from the WRF simulation at $t=59.0 \mathrm{~h}$ (the selected time is chosen to match the one in B1). Experiment $\mathrm{C} 2$ is initialized with a vorticity profile extracted a few hours later $(t=63.5 \mathrm{~h})$. In $\mathrm{C} 1$, until $t_{\mathrm{NDBV}}=12.0 \mathrm{~h}$ the flow does not develop obvious $m=2$ asymmetry (larger $e$-folding time relative to experiment B2). Comparing this outcome with $\mathrm{C} 2$, it appears that an $m=2$ asymmetry develops much faster in the latter experiment ( $e$-folding time of $4.1 \mathrm{~h}$ ). Nevertheless, here the instability remains much slower than that in experiment $\mathrm{A} 2$ ( $e$-folding time of $0.7 \mathrm{~h}$ ). It is therefore suggested that at upper levels, the $m=2$ instability may not be sufficiently intense to develop an equivalent asymmetric radial flow pattern. Note that the NDBV framework does not support the excitation of an exponential $m=1$ instability. As a result, the $m=1$ pattern (see Fig. 15) does not emerge in these experiments.

In summary, the above results suggest that the absence of a second (and faster) period of $C_{\text {in }}$ decline in the middle and upper levels might be attributed to the 


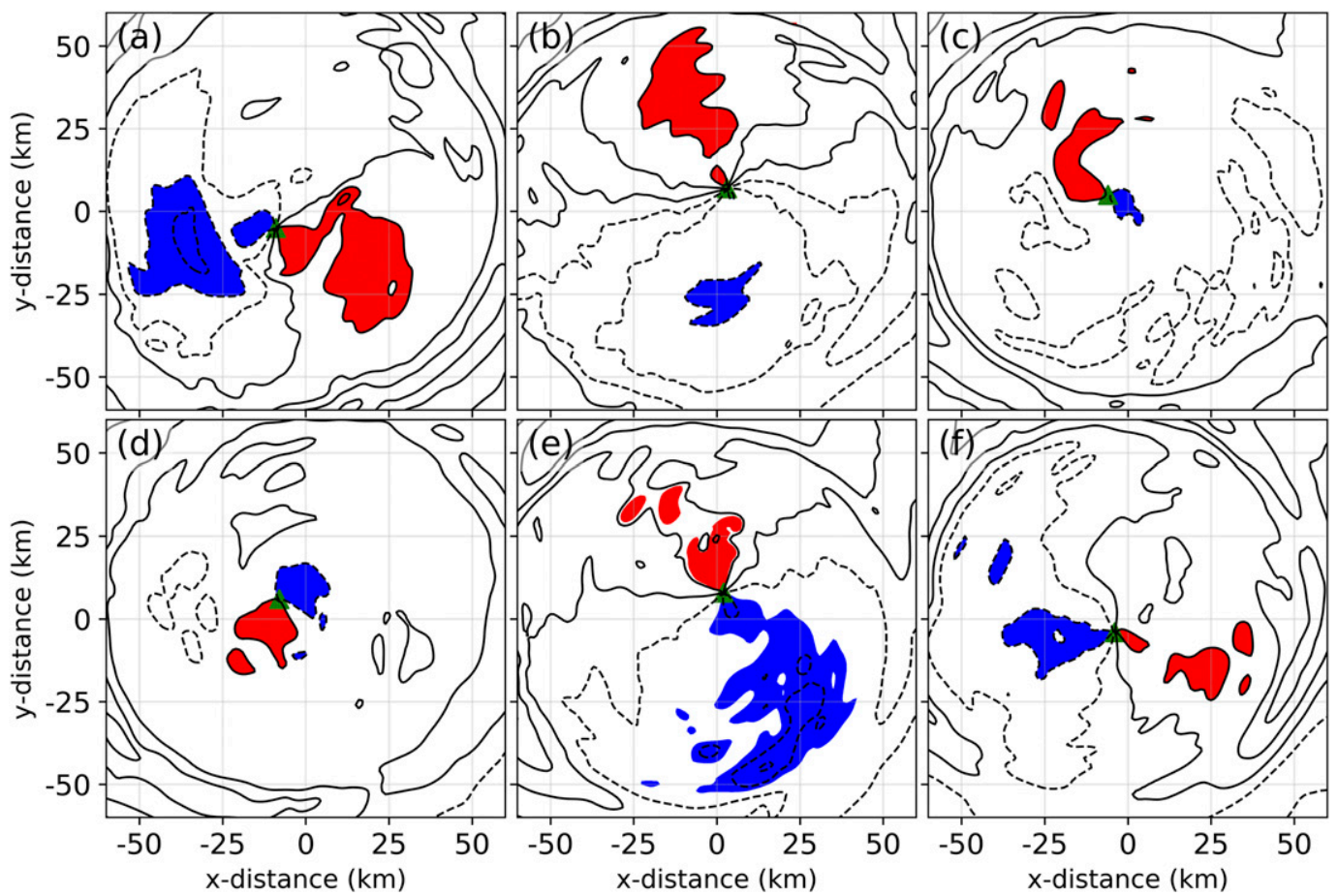

FIG. 15. As in Fig. 14, but for the upper levels $(7.5 \leq z \leq 11 \mathrm{~km})$. Green triangles mark the upper-level center of the simulated Wilma. (e) The color shading is produced with a different set of contour levels.

inability of a dominant $m=2$ type-2 instability to be sustained (and thus there is no persistent dominant $m=$ 2 radial flow asymmetry). Physically, this difference in evolution might be accounted for by the differences in the moat width as per the linear stability analysis: at the middle and upper levels the moat width is much larger than that at the lower levels (in addition to the outer eyewall being weaker at the higher levels). Specifically, the midlevel moat width is around $30 \mathrm{~km}$ (solid red curve; Fig. 10) while the low-level moat width is about $15 \mathrm{~km}$ (dotted blue curve; Fig. 10). According to the linear stability analysis, a type- 2 instability is expected to be much faster and most likely to dominate the instability at the lower levels. It should also be remarked that the differences in the moat width at different heights are to be expected as the eyewalls in TCs often exhibit profound differences in outward slope. In the case of the simulated Wilma, the outward slope of the outer eyewall is greater than that of the inner eyewall (figure not shown). This behavior has also been documented in other TC simulations (e.g., Zhou and Wang 2011, their Figs. 11 and 13; Wang et al. 2016, their Fig. 3; Tsujino et al. 2017, their Figs. 10b and 18b; Zhang et al. 2017, their Fig. 6) and in real TC observations (e.g., Zhao et al. 2016, their Fig. 3). Stern and Nolan (2009) and Stern et al. (2014) found that the outward slopes of the radius of maximum tangential winds (RMW) and the outward slopes of the eyewall absolute angular momentum surfaces systematically increase with the sizes of the RMW. Since an outer eyewall is by definition at a greater radius than the corresponding inner eyewall, the outward slope of the outer eyewall is larger. Similar findings were also reported by Hazelton and Hart (2013).

\section{e. Thermodynamic effect}

Another effect due to the mixing of air induced by barotropic instability of the vortex is the modification of its thermodynamic structure. In a TC with a single eyewall, Persing and Montgomery (2003) argued that mixing between the eye and the eyewall, perhaps induced by the nonlinear stirring from VRWs at the inner edge of the eyewall, can stir higher $\theta_{e}$ from the eye to the eyewall. This stirring can intensify a TC to beyond its maximum potential intensity (Emanuel 1986, 1988, 1995; Bister and Emanuel 1998). A similar conclusion was reached by Hendricks and Schubert (2009) on the basis that a maximum of $\theta_{e}$ at the lower levels has been observed in the hurricane eye (Eastin et al. 2002a,b). On the other hand, in an axisymmetric framework, Bryan and Rotunno (2009) found that the total magnitude of entropy transport between the eye and eyewall is negligible in their simulated TCs. Based on the facts that the simulated Wilma is clearly 
(a)
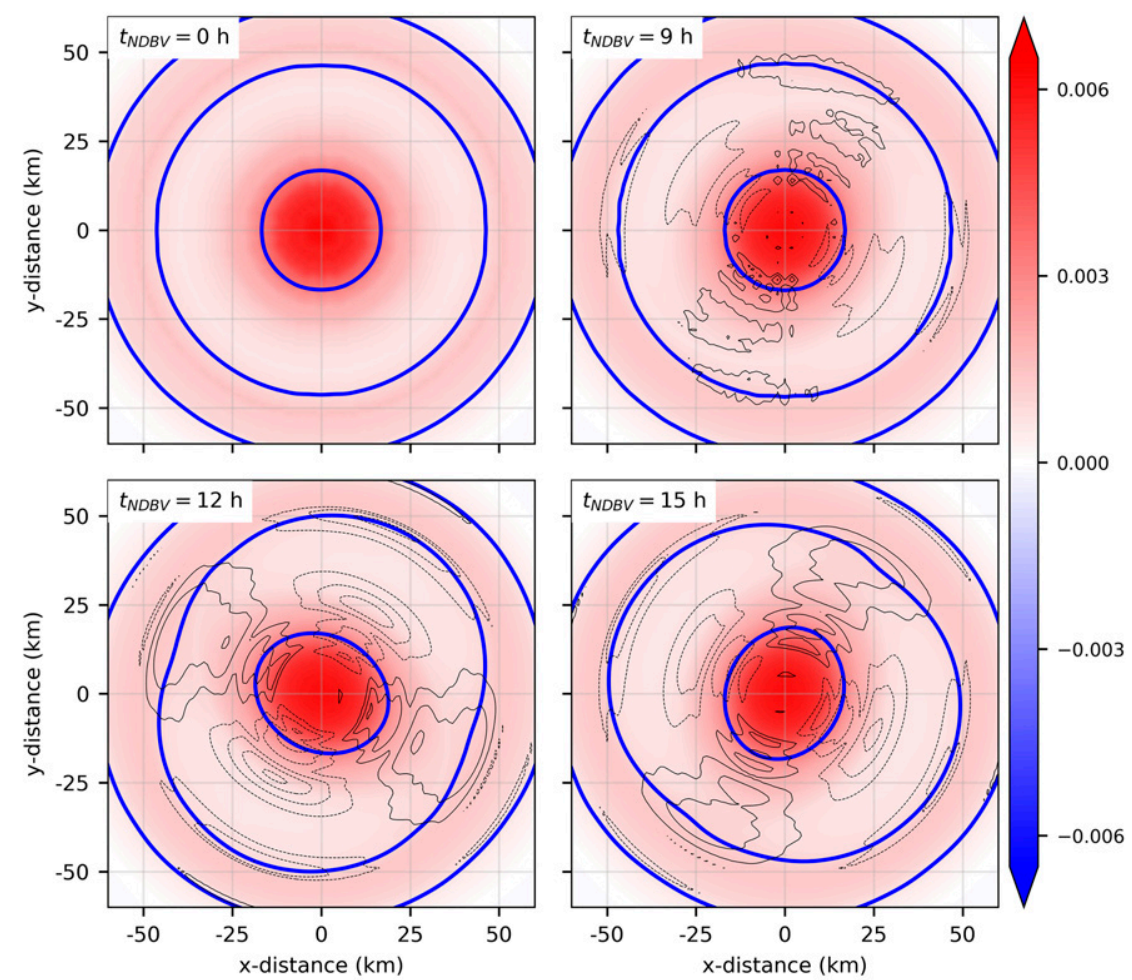

(b)

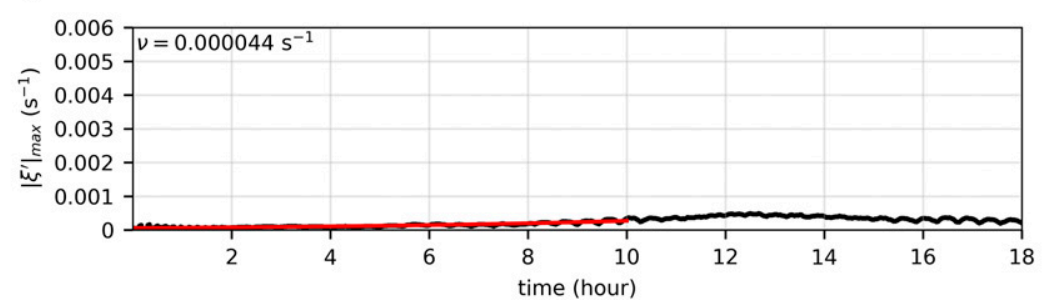

FIG. 16. NDBV experiment B1. (a) Relative vorticity $\zeta$ (shading) and its perturbation $\zeta^{\prime}$ (black contours) at the indicated experiment times. Positive values (solid) are contoured from $\zeta^{\prime}=1.5 \times 10^{-4} \mathrm{~s}^{-1}$ by $1.5 \times 10^{-4} \mathrm{~s}^{-1}$ while negative values (dashed) are contoured from $\zeta^{\prime}=-1.5 \times 10^{-4} \mathrm{~s}^{-1}$ by $-1.5 \times 10^{-4} \mathrm{~s}^{-1}$. The $m \geq 5$ patterns of the $\zeta^{\prime}$ field are filtered out. The blue contours of $\partial^{2} \zeta / \partial r^{2}=0$ outline the shape of the outer eyewall, the moat, and the inner eyewall. The distance is with respect to the center of the vortex. (b) The time series of the maximum absolute values of the perturbation relative vorticity $\left|\zeta^{\prime}\right|_{\max }$ (black dots). The red curve is the exponential fit for the corresponding period and $\nu$ is the corresponding growth rate. The abscissa is the experiment time.

asymmetric and that it has a double eyewall (i.e., three types of barotropic instability can be excited in the three regions of the vortex), the asymmetric mixing of $\theta_{e}$ can be significant. Therefore, it is of interest to explore how the instability across the moat can modify the thermodynamic structure of the simulated Wilma.

Figure 18 presents the evolution of low-level equivalent potential temperature $\theta_{e}$, including the remarkable elliptic elongation of the high $\theta_{e}$ patch at the inner eyewall and the eye (the inner $\theta_{e}$ patch). By comparing this figure with Fig. 3, it is clear that $\theta_{e}$ in the moat region is significantly lower than that in the inner eyewall. ${ }^{12}$ By referring to Figs. 18 and 4, it can be expected that the $m=2$ pattern of the low-level radial flow would dilute the inner $\theta_{e}$ patch in a manner similar to the dilution of the inner vorticity patch. Using the same

\footnotetext{
${ }^{12}$ From the thermodynamics viewpoint, a strengthening outer eyewall will lead to an enhanced warm core, and thereby increasing potential temperature. Assuming the water vapor mixing ratio does not decrease substantially in the moat during this time, it is expected that $\theta_{e}$ within the moat will increase. The dilution (or mixing) between the eyewalls and the moat, associated with type-2 instability, may accelerate the warming process of the moat.
} 
(a)
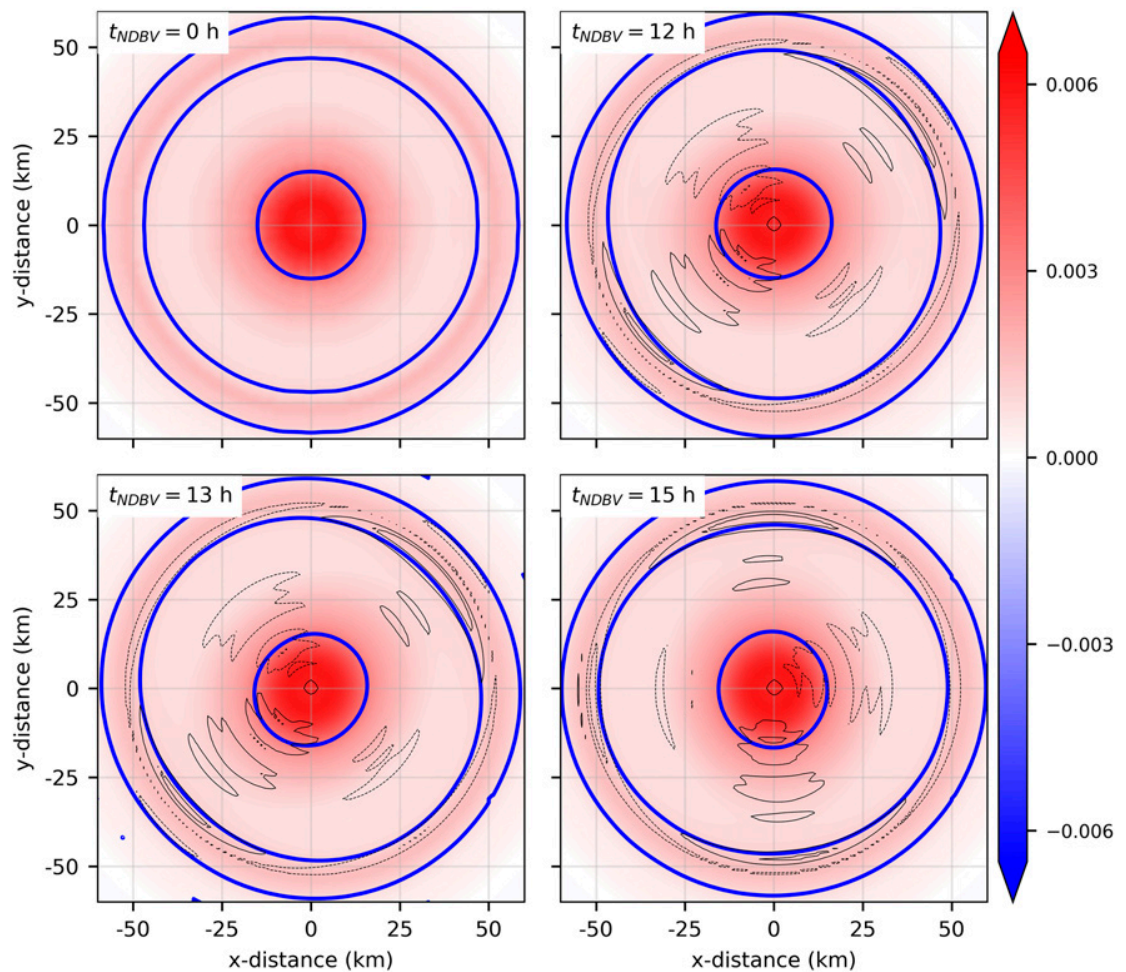

(b)

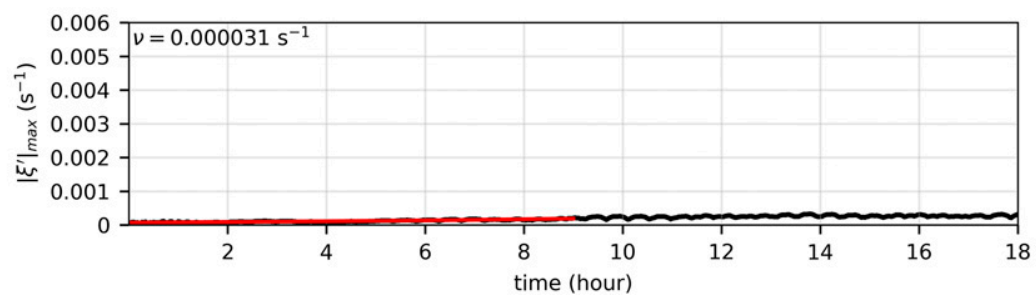

FIG. 17. As in Fig. 16, but for NDBV experiment B2.

calculation region for $C_{\text {in }}$, the time series of the area integral of equivalent potential temperature of the inner vorticity patch $\theta_{e, \text { sum }}=\iint_{A} \theta_{e} d A$ is obtained (Fig. 19). It is clear that after SEF $(t \approx 42.0 \mathrm{~h}), \theta_{e \text { sum }}$ remains roughly unchanged, indicating that the moist entropy supply to the inner eyewall is still insignificantly intercepted. Later, at $t=60.5 \mathrm{~h}$ which is $1 \mathrm{~h}$ after the change of the low-level dominant radial flow pattern, $\theta_{e \text {,sum }}$ starts decreasing substantially at the lower levels (the blue curve), suggesting that the dilution effect discussed in section 4 also acts on the $\theta_{e}$ field. In other words, the outward-breaking VRWs at the outer edge of the inner eyewall, induced by the type- 2 instability, mix the air with lower $\theta_{e}$ from the moat region into the inner eyewall. This weakening $\theta_{e \text {,sum }}$ means the decrease in the supply of moist entropy to the inner eyewall, possibly contributing to its decay. Thus the type- 2 instability may impose a detrimental effect on the intensity of the simulated Wilma by modifying its thermodynamic structure at the lower levels.

In passing, we mention that similar to the evolution of low-level $C_{\text {in }}$, there is also a time period in which the decrease in $\theta_{e \text { sum }}$ almost pauses (from $t=64.0$ to $66.0 \mathrm{~h}$ ), and another period in which the declining rate of $\theta_{e \text {,sum }}$ is much smaller (from $t=67.5$ to $69.0 \mathrm{~h}$ ). As discussed in section $5 \mathrm{c}$, these two periods are marked by the dominant radial flow pattern changing back from wavenumber $m=2$ to $m=1$ (i.e., the normal strain pattern of radial flow becomes nondominant). ${ }^{13}$

\footnotetext{
${ }^{13}$ Although the temporary change of dominant radial flow pattern is a qualitatively reasonable explanation for these pauses, the definite underlying mechanism requires further investigation.
} 

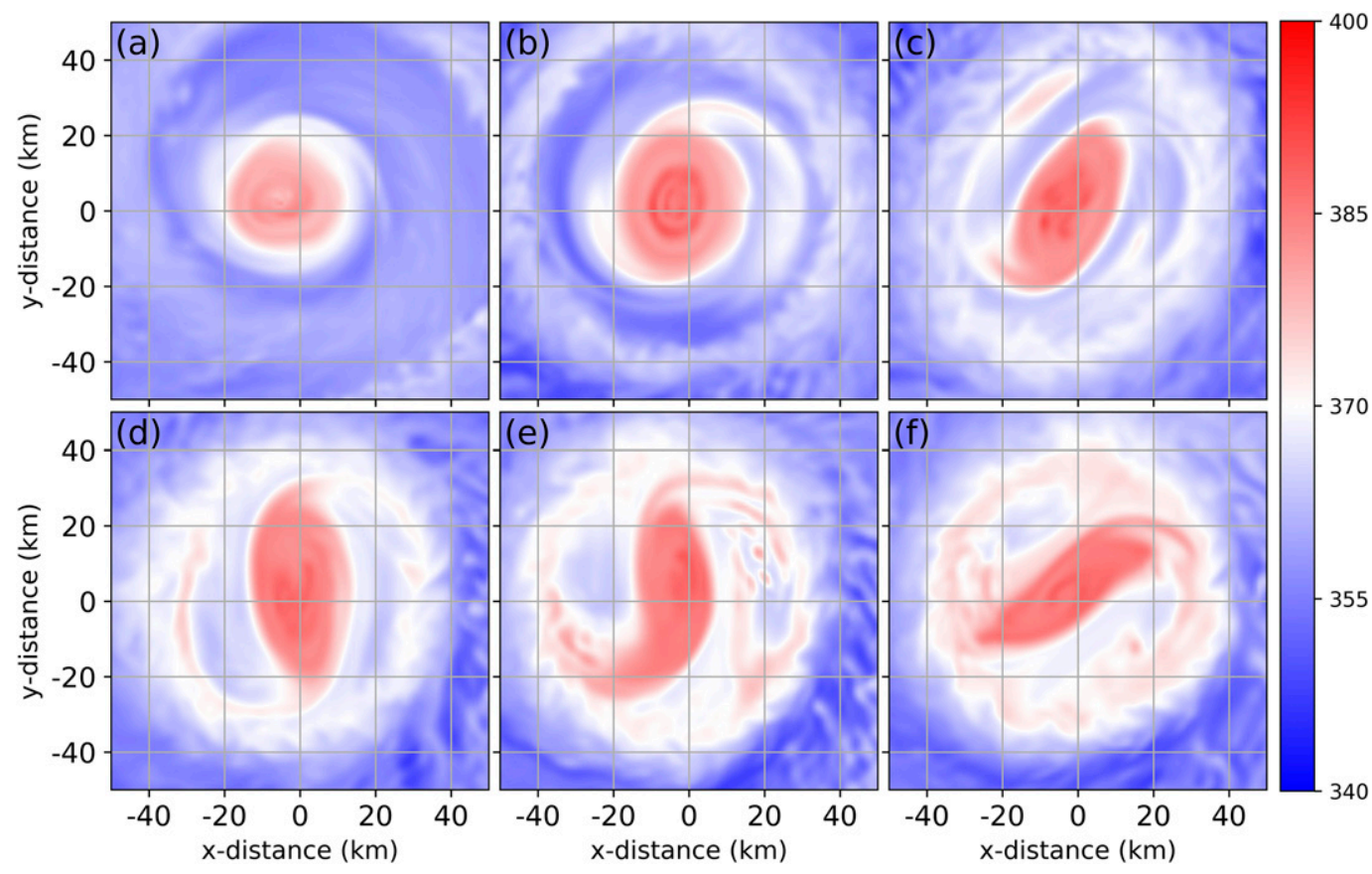

FIG. 18. Equivalent potential temperature $\theta_{e}(\mathrm{~K})$ of the simulated Wilma averaged over the lower levels $(0.8 \leq z \leq 2.5 \mathrm{~km})$ at $t=$ (a) 42 , (b) 54 , (c) 59.5 , (d) 62 , (e) 64 , and (f) $66 \mathrm{~h}$. The distance is with respect to the center of the innermost simulation domain.

In addition, the trends in the mid- and upper-level $\theta_{e, \text { sum }}$ (red and green curves of Fig. 19) indicate no substantial decrease with time, unlike the lower levels. The reason is similar to that of the evolution of $C_{\mathrm{in}}$, discussed in section $5 \mathrm{~d}$.

It should be cautioned that the thermodynamic effect described above is incomplete because the changes in vertical motion or convection, which are out of the scope of this paper, can have substantial effects on the inner eyewall strength. For instance, it is possible that the low-level radial outflow pair during the occurrence of type-2 instability induces nonnegligible downdrafts (if the divergence of outflow pair exceeds the convergence of inflow pair), which counteract the convective updrafts within the inner eyewall. The resultant weaker updrafts may yield a weaker latent heat release and a weaker warm core, unfavorable for the maintenance of the strength of the TC vortex. In addition, both ABL physics and the effect of diabatic heating are excluded in the theoretical framework of the five-region model developed from the work of KSM00. Therefore, the effects of the moist entropy distribution and convection in a TC with a double eyewall on type- 2 instability could not be identified in the current paper. Further work would be required to shed light on the interaction between the type- 2 instability, convection, and the thermodynamic structure of a TC with a double eyewall.

\section{Conclusions}

In TCs with multiple eyewalls, the problem of how the inner eyewall dissipates remains an open question. Some recent radar observations indicate that the inner

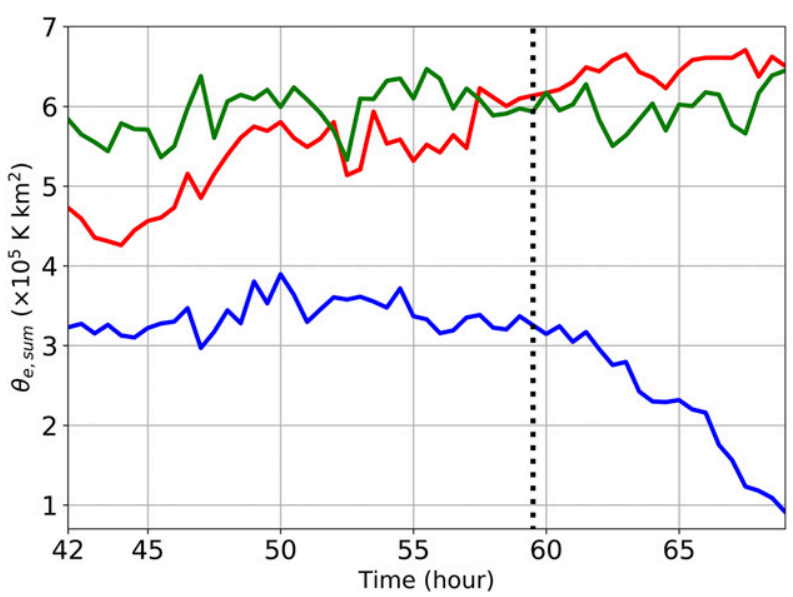

FIG. 19. Time series of the sum of equivalent potential temperature $\left(\mathrm{K} \mathrm{km}^{2}\right)$ of the inner vorticity patch $\theta_{e, \text { sum }}$ (the area integral of $\theta_{e}$ within the inner vorticity patch) calculated from the WRF simulation output, averaged over the lower levels (blue), middle levels (red), and upper levels (green). The black dotted line marks the start of the long-lasting period in which the dominant low-level radial flow pattern is wavenumber 2 . The abscissa is the simulation time since 0000 UTC 18 Oct 2005. 
eyewalls become elliptical prior to their dissipation. It is conjectured that barotropic instability across the moat (see KSM00; aka type-2 barotropic instability) may play a role in this process. To investigate this problem, a 72-h high-resolution WRF simulation of Hurricane Wilma (2005) was performed. The simulation reproduced reasonably well the track, initial spinup, RI, peak intensity, and subsequent weakening of Wilma. However, the simulated track is about $185 \mathrm{~km}$ too far to the north-northeast of the best track at the end of the 72-h simulation. Of importance is that the simulation captures Wilma's first ERC, with similar duration.

The simulation results indicate that the low-level eyewall became more and more elliptical for a $10-\mathrm{h}$ period prior to its dissipation. During this period, the radial flow changed from a dominant pattern with wavenumber $m=1$ to $m=2$, which is suggestive of the emergence of a type- 2 barotropic instability mode with $m=2$. To ascertain this process in a TC with a double eyewall, a linear stability analysis in the context of a $2 \mathrm{D}$ nondivergent barotropic vorticity model was performed. Specifically, the four-region model of KSM00 was extended to a five-region model and a type- 2 barotropic instability was obtained. The linear results are supported by nonlinear computations using a nonlinear nondivergent barotropic vorticity (NDBV) model. By initializing the NDBV model with the axisymmetric vorticity profiles from the WRF simulation before the wavenumber changed from $m=1$ to $m=2$, it was confirmed that the initialized vorticity profiles were susceptible to the $m=2$ type- 2 barotropic instability.

Of particular importance is the finding of how this instability results in the dissipation of the inner eyewall. The proposed mechanism is presented in a schematic diagram (Fig. 20). The radial flow associated with the dominant $m=2$ mode of a type-2 barotropic instability has a normal strain pattern. If this pattern lasts for a long time, then the outer part of the inner vorticity patch would be greatly diluted (mainly in the form of shrinkage of the inner vorticity patch)-the inner eyewall significantly decays-because of the rapid filamentation at the two ends of the elliptically elongated inner vorticity patch associated with the cyclonic differential rotation (with respect to the moat), as well as the intrusion of low- $\zeta$ air from the moat which is associated with the radial inflow pair. To provide more direct evidence, the time series of $C_{\text {in }}$ within the inner vorticity column was produced. It was clearly shown that, apart from the persistent decrease in $C_{\text {in }}$ shortly after SEF (which could have resulted from the establishment of the outer eyewall), there was a period in which the lowlevel $C_{\text {in }}$ decreased more significantly. The time of occurrence of this period matches very well the period

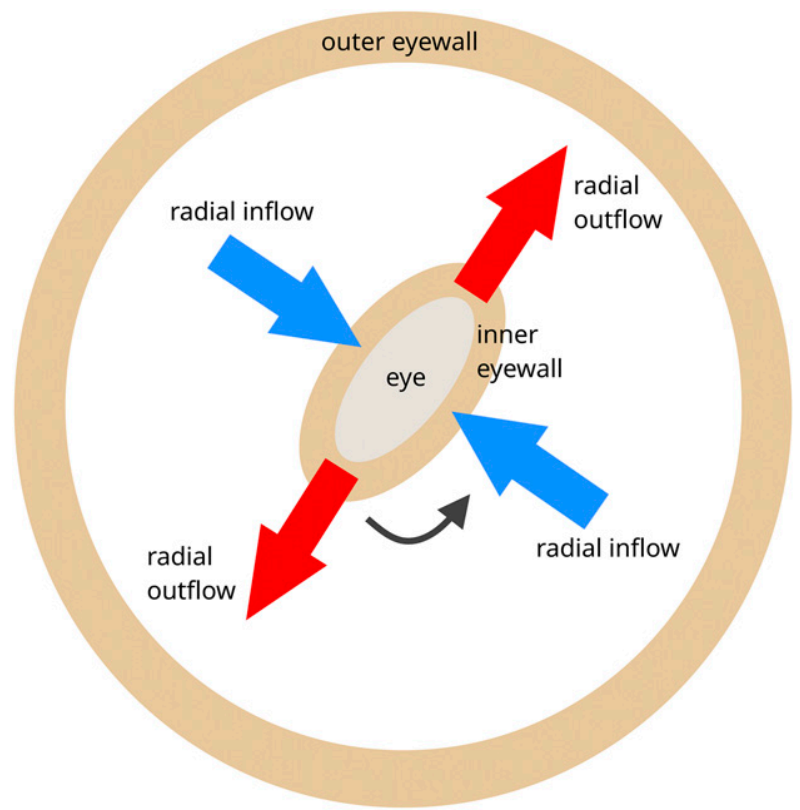

FIG. 20. A schematic diagram of the dilution of vorticity of the inner eyewall and the eye by mixing with the lower-vorticity air in the moat, due to the wavenumber- 2 radial flow pattern associated with type- 2 barotropic instability. Red arrows indicate the radial outflow pair elliptically elongating the inner vorticity patch while blue arrows indicate the radial inflow pair transporting low $\zeta$ into the inner vorticity patch. The dark gray curved arrow indicates the cyclonic rotation (relative to the moat) of the inner vorticity patch.

in which the dominant pattern of low-level radial flow changed from $m=1$ to $m=2$. This finding supports the idea that type-2 barotropic instability resulted in a significant dilution of the low-level inner vorticity patch in the simulated Wilma.

To find out the reason why there was no such second period of significant decrease in $C_{\text {in }}$ at other levels, the corresponding radial flow evolutions were studied and another two sets of NDBV experiments were performed. At the middle levels, it was shown that $m=2$ type-2 barotropic instability was excited but short lived. The corresponding set of NDBV experiments revealed that the midlevel vorticity profiles were only weakly unstable. At the upper levels, it was demonstrated that the instability was even weaker. Although it became stronger at later times (NDBV experiment $\mathrm{C} 2$ ), it was not intense enough to become dominant (an $m=1$ pattern was persistent). The difference in evolution among these three levels could be explained by the nature of type-2 barotropic instability which has a threshold of moat width for excitation. Given that the outward slope of the outer eyewall is greater than that of the inner eyewall, the moat width increases with altitude (as shown in Fig. 10). Consequently, with increasing height, the instability has a weaker and weaker growth rate. 
Another finding is that the type- 2 instability can also have detrimental effects on the intensity of the simulated Wilma by modifying the low-level thermodynamic structure of the storm through the mixing of lower $\theta_{e}$ into the inner eyewall from the moat region. However, given that convection was not included in our analyses and both $\mathrm{ABL}$ physics and diabatic heating were excluded in the fiveregion model, the study of the interaction between a type- 2 barotropic instability and moist entropy and deep convection in a double-eyewall TC remains incomplete. Future work is planned to shed light on this subject.

In closing, it is cautioned that the presented analyses focused on exploring a possible pathway to the dissipation of the inner eyewall without taking the frictional boundary layer into consideration. However, boundary layer effects could play an important role in the inner eyewall dissipation (Huang et al. 2012; Kepert 2013; Montgomery et al. 2014; Kepert and Nolan 2014; Tsujino et al. 2017; Zhang and Perrie 2018). The extent to which the possible contribution from type-2 instability remains significant (or is modified) in the presence of a boundary layer will be explored and reported in a follow-up paper.

Acknowledgments. The GFDL hurricane model data used for the simulation were kindly provided by Timothy Marchok at NOAA/GFDL. We thank Peter Bartello for providing the nondivergent barotropic spectral model. We also thank Michael T. Montgomery and two anonymous reviewers for their constructive comments that improved the manuscript. This research reported here is supported by the Natural Sciences and Engineering Research Council of Canada (NSERC)/Hydro-Québec Industrial Research Chair (IRC) program. The simulation was performed on the supercomputer Graham supported by Compute Canada (www.computecanada.ca).

\section{APPENDIX}

\section{The Matrix $M *$ and Solving the Dimensionless Eigenvalue Problem}

This appendix provides the details of solving the eigenvalue problem (9) and of the associated matrix operator $\mathbf{M}^{*}$.

The expression of $\mathbf{M}^{*}$ is given as

$$
\mathbf{M}^{*}=\left(\begin{array}{llll}
M_{1,1}^{*} & M_{1,2}^{*} & M_{1,3}^{*} & M_{1,4}^{*} \\
M_{2,1}^{*} & M_{2,2}^{*} & M_{2,3}^{*} & M_{2,4}^{*} \\
M_{3,1}^{*} & M_{3,2}^{*} & M_{3,3}^{*} & M_{3,4}^{*} \\
M_{4,1}^{*} & M_{4,2}^{*} & M_{4,3}^{*} & M_{4,4}^{*}
\end{array}\right),
$$

where

$$
\begin{aligned}
& M_{1,1}^{*}=m \frac{\zeta_{0}}{\zeta_{3}}+\left(\frac{\zeta_{1}-\zeta_{0}}{\zeta_{3}}\right) \\
& M_{1,2}^{*}=\left(\frac{\zeta_{1}-\zeta_{0}}{\zeta_{3}}\right)\left(\frac{r_{0}}{r_{1}}\right)^{m}, \\
& M_{1,3}^{*}=\left(\frac{\zeta_{1}-\zeta_{0}}{\zeta_{3}}\right)\left(\frac{r_{0}}{r_{2}}\right)^{m}, \\
& M_{1,4}^{*}=\left(\frac{\zeta_{1}-\zeta_{0}}{\zeta_{3}}\right)\left(\frac{r_{0}}{r_{3}}\right)^{m} \text {, } \\
& M_{2,1}^{*}=-\frac{\zeta_{1}}{\zeta_{3}}\left(\frac{r_{0}}{r_{1}}\right)^{m} \\
& M_{2,2}^{*}=m\left[\frac{\zeta_{1}}{\zeta_{3}}-\left(\frac{\zeta_{1}-\zeta_{0}}{\zeta_{3}}\right)\left(\frac{r_{0}}{r_{1}}\right)^{2}\right]-\frac{\zeta_{1}}{\zeta_{3}}, \\
& M_{2,3}^{*}=-\frac{\zeta_{1}}{\zeta_{3}}\left(\frac{r_{1}}{r_{2}}\right)^{m} \\
& M_{2,4}^{*}=-\frac{\zeta_{1}}{\zeta_{3}}\left(\frac{r_{1}}{r_{3}}\right)^{m} \\
& M_{3,1}^{*}=\left(\frac{r_{0}}{r_{2}}\right)^{m} \\
& M_{3,2}^{*}=\left(\frac{r_{1}}{r_{2}}\right)^{m} \\
& M_{3,3}^{*}=m\left[\frac{\zeta_{1}}{\zeta_{3}}\left(\frac{r_{1}}{r_{2}}\right)^{2}-\left(\frac{\zeta_{1}-\zeta_{0}}{\zeta_{3}}\right)\left(\frac{r_{0}}{r_{2}}\right)^{2}\right]+1 \\
& M_{3,4}^{*}=\left(\frac{r_{2}}{r_{3}}\right)^{m} \text {, } \\
& M_{4,1}^{*}=-\left(\frac{r_{0}}{r_{3}}\right)^{m} \text {, } \\
& M_{4,2}^{*}=-\left(\frac{r_{1}}{r_{3}}\right)^{m} \text {, }
\end{aligned}
$$$$
M_{4,3}^{*}=-\left(\frac{r_{2}}{r_{3}}\right)^{m} \text {, }
$$$$
M_{4,4}^{*}=m\left[1-\left(\frac{r_{2}}{r_{3}}\right)^{2}+\frac{\zeta_{1}}{\zeta_{3}}\left(\frac{r_{1}}{r_{3}}\right)^{2}-\left(\frac{\zeta_{1}-\zeta_{0}}{\zeta_{3}}\right)\left(\frac{r_{0}}{r_{3}}\right)^{2}\right]-1 .
$$

The eigenvalue problem (9) can be solved by solving the following determinant equation:

$$
\left|\mathbf{M}^{*}-\nu^{*} \mathbf{I}\right|=0,
$$


where $\mathbf{I}$ is a $4 \times 4$ identity matrix. For given values of $m$, $r_{0} / r_{1}, r_{1} / r_{2}, r_{2} / r_{3}, \zeta_{0} / \zeta_{1}$, and $\zeta_{1} / \zeta_{3}$, solving (A2) gives four values of the dimensionless frequency $\nu^{*}=2 \nu / \zeta_{3}$. The most unstable value from these four roots is chosen for each $m$. Then, the mode with $m$ having the largest corresponding dimensionless growth rate $\nu_{i}^{*}$ is identified as the most unstable mode (the dominant mode). Note that in this paper, $\zeta_{0} / \zeta_{1}$ is fixed as 0.88 for the first set of linear stability analysis diagrams (Figs. 6 and 7). This chosen value is based on our simulation of Hurricane Wilma (2005) at $t=59.0 \mathrm{~h}$ during the double-eyewall stage.

\section{REFERENCES}

Abarca, S. F., and M. T. Montgomery, 2013: Essential dynamics of secondary eyewall formation. J. Atmos. Sci., 70, 3216-3230, https://doi.org/10.1175/JAS-D-12-0318.1.

_ , and _ 2014: Departures from axisymmetric balance dynamics during secondary eyewall formation. J. Atmos. Sci., 71, 3723-3738, https://doi.org/10.1175/JAS-D-14-0018.1.

Barnes, G. M., E. J. Zipser, D. Jorgensen, and F. Marks Jr., 1983: Mesoscale and convective structure of a hurricane rainband. J. Atmos. Sci., 40, 2125-2137, https://doi.org/10.1175/ 1520-0469(1983)040<2125:MACSOA > 2.0.CO;2.

Bartello, P., and T. Warn, 1996: Self-similarity of decaying twodimensional turbulence. J. Fluid Mech., 326, 357-372, https:// doi.org/10.1017/S002211209600835X.

Bister, M., and K. A. Emanuel, 1998: Dissipative heating and hurricane intensity. Meteor. Atmos. Phys., 65, 233-240, https:// doi.org/10.1007/BF01030791

Black, M. L., and H. E. Willoughby, 1992: The concentric eyewall cycle of Hurricane Gilbert. Mon. Wea. Rev., 120, 947-957, https://doi.org/10.1175/1520-0493(1992)120<0947:TCECOH > 2.0.CO;2.

Bryan, G. H., and R. Rotunno, 2009: The influence of near-surface, high-entropy air in hurricane eyes on maximum hurricane intensity. J. Atmos. Sci., 66, 148-158, https://doi.org/10.1175/ 2008JAS2707.1.

Chen, H., D.-L. Zhang, and J. Carton, 2011: On the rapid intensification of Hurricane Wilma (2005). Part I: Model prediction and structural changes. Wea. Forecasting, 26, 885-901, https://doi.org/ 10.1175/WAF-D-11-00001.1.

Chou, M.-D., and M. J. Suarez, 1994: An efficient thermal infrared radiation parameterization for use in general circulation models. NASA Tech. Memo. 104606, Vol. 3, 85 pp.

Didlake, A. C., Jr., G. M. Heymsfield, P. D. Reasor, and S. R. Guimond, 2017: Concentric eyewall asymmetries in Hurricane Gonzalo (2014) observed by airborne radar. Mon. Wea. Rev., 145, 729-749, https://doi.org/10.1175/MWR-D-16-0175.1.

Eastin, M. D., P. G. Black, and W. M. Gray, 2002a: Flight-level thermodynamic instrument wetting errors in hurricanes. Part I: Observations. Mon. Wea. Rev., 130, 825-841, https://doi.org/ 10.1175/1520-0493(2002)130<0825:FLTIWE > 2.0.CO;2.

,-- , and $-2002 \mathrm{~b}$ : Flight-level thermodynamic instrument wetting errors in hurricanes. Part II: Implications. Mon. Wea. Rev., 130, 842-851, https://doi.org/10.1175/1520-0493(2002) 130<0842:FLTIWE $>2.0$. CO;2.

Emanuel, K. A., 1986: An air-sea interaction theory for tropical cyclones. Part I: Steady-state maintenance. J. Atmos. Sci., 43,
585-604, https://doi.org/10.1175/1520-0469(1986)043<0585: AASITF $>2.0 . \mathrm{CO} ; 2$.

, 1988: The maximum intensity of hurricanes. J. Atmos. Sci., 45, 1143-1155, https://doi.org/10.1175/1520-0469(1988)045<1143: $\mathrm{TMIOH}>2.0 . \mathrm{CO} ; 2$.

1995: Sensitivity of tropical cyclones to surface exchange coefficients and a revised steady-state model incorporating eye dynamics. J. Atmos. Sci., 52, 3969-3976, https://doi.org/ 10.1175/1520-0469(1995)052<3969:SOTCTS > 2.0.CO;2.

Gadoury, J., 2012: Impact of numerical grid spacing and time step size on vortex Rossby waves in secondary eyewall formation in a simulation of hurricane Wilma (2005). M.S. thesis, Dept. of Atmospheric and Oceanic Sciences, McGill University, 115 pp.

Hawkins, J. D., and M. Helveston, 2008: Tropical cyclone multiple eyewall characteristics. 28th Conf. on Hurricanes and Tropical Meteorology, Orlando, FL, Amer. Meteor. Soc., 14B.1, https://ams.confex.com/ams/28Hurricanes/webprogram/ Paper138300.html.

Hazelton, A. T., and R. E. Hart, 2013: Hurricane eyewall slope as determined from airborne radar reflectivity data: Composites and case studies. Wea. Forecasting, 28, 368-386, https://doi.org/ 10.1175/WAF-D-12-00037.1.

Hendricks, E. A., and W. H. Schubert, 2009: Transport and mixing in idealized barotropic hurricane-like vortices. Quart. J. Roy. Meteor. Soc., 135, 1456-1470, https://doi.org/10.1002/qj.467.

Hoskins, B. J., M. E. McIntyre, and A. W. Robertson, 1985: On the use and significance of isentropic potential vorticity maps. Quart. J. Roy. Meteor. Soc., 111, 877-946, https://doi.org/ 10.1002/qj.49711147002.

Houze, R. A., Jr., and Coauthors, 2006: The Hurricane Rainband and Intensity Change Experiment: Observations and modeling of Hurricanes Katrina, Ophelia, and Rita. Bull. Amer. Meteor. Soc., 87, 1503-1521, https://doi.org/10.1175/ BAMS-87-11-1503.

, S. S. Chen, B. F. Smull, W.-C. Lee, and M. M. Bell, 2007: Hurricane intensity and eyewall replacement. Science, 315, 1235-1239, https://doi.org/10.1126/science.1135650.

Huang, Y.-H., M. T. Montgomery, and C.-C. Wu, 2012: Concentric eyewall formation in Typhoon Sinlaku (2008). Part II: Axisymmetric dynamical processes. J. Atmos. Sci., 69, 662-674, https://doi.org/10.1175/JAS-D-11-0114.1.

Janjić, Z. I., 1990: The step-mountain coordinate: Physical package. Mon. Wea. Rev., 118, 1429-1443, https://doi.org/10.1175/ 1520-0493(1990)118<1429:TSMCPP>2.0.CO;2.

_ 1994: The step-mountain eta coordinate model: Further developments of the convection, viscous sublayer, and turbulence closure schemes. Mon. Wea. Rev., 122, 927-945, https://doi.org/10.1175/1520-0493(1994)122<0927:TSMECM> 2.0.CO;2.

, 1996: The surface layer in the NCEP Eta Model. Preprints, 11th Conf. on Numerical Weather Prediction, Norfolk, VA, Amer. Meteor. Soc., 354-355.

_ 2000: Comments on "Development and evaluation of a convection scheme for use in climate models." J. Atmos. Sci., 57, 3686, https://doi.org/10.1175/1520-0469(2000)057<3686: CODAEO $>2.0 . \mathrm{CO} ; 2$.

— 2002: Nonsingular implementation of the Mellor-Yamada level 2.5 scheme in the NCEP Meso Model. NCEP Office Note 437, $61 \mathrm{pp}$.

Judt, F., and S. S. Chen, 2010: Convectively generated potential vorticity in rainbands and formation of the secondary eyewall in Hurricane Rita of 2005. J. Atmos. Sci., 67, 3581-3599, https:// doi.org/10.1175/2010JAS3471.1. 
Kepert, J. D., 2013: How does the boundary layer contribute to eyewall replacement cycles in axisymmetric tropical cyclones? J. Atmos. Sci., 70, 2808-2830, https://doi.org/10.1175/ JAS-D-13-046.1.

— , and D. S. Nolan, 2014: Reply to "Comments on 'How does the boundary layer contribute to eyewall replacement cycles in axisymmetric tropical cyclones?"' J. Atmos. Sci., 71, 46924704, https://doi.org/10.1175/JAS-D-14-0014.1.

Kimberlain, T. B., E. S. Blake, and J. P. Cangialosi, 2016: Hurricane Patricia (EP202015) 20-24 October 2015. National Hurricane Center Rep., 32 pp., https://www.nhc.noaa.gov/data/tcr/ EP202015_Patricia.pdf.

Kossin, J. P., W. H. Schubert, and M. T. Montgomery, 2000: Unstable interactions between a hurricane's primary eyewall and a secondary ring of enhanced vorticity. J. Atmos. Sci., 57, 3893-3917, https://doi.org/10.1175/1520-0469(2001)058<3893: UIBAHS $>2.0$. CO;2.

Kuo, H.-C., L.-Y. Lin, C.-P. Chang, and R. T. Williams, 2004: The formation of concentric vorticity structures in typhoons. J. Atmos. Sci., 61, 2722-2734, https://doi.org/10.1175/ JAS3286.1.

— - W. H. Schubert, C.-L. Tsai, and Y.-F. Kuo, 2008: Vortex interactions and barotropic aspects of concentric eyewall formation. Mon. Wea. Rev., 136, 5183-5198, https://doi.org/ 10.1175/2008MWR2378.1.

Lawrence, M. B., and J. M. Gross, 1989: Atlantic hurricane season of 1988. Mon. Wea. Rev., 117, 2248-2259, https://doi.org/10.1175/ 1520-0493(1989)117<2248:AHSO > 2.0.CO;2.

Martinez, Y., G. Brunet, M. K. Yau, and X. Wang, 2011: On the dynamics of concentric eyewall genesis: Space-time empirical normal modes diagnosis. J. Atmos. Sci., 68, 457-476, https:// doi.org/10.1175/2010JAS3501.1.

McNoldy, B. D., 2004: Triple eyewall in Hurricane Juliette. Bull. Amer. Meteor. Soc., 85, 1663-1666, https://doi.org/10.1175/ BAMS-85-11-1663.

__, 2012: Tropical cyclone radar loops. University of Miami, http://andrew.rsmas.miami.edu/bmcnoldy/tropics/radar/.

Menelaou, K., M. K. Yau, and Y. Martinez, 2012: On the dynamics of the secondary eyewall genesis in Hurricane Wilma (2005). Geophys. Res. Lett., 39, L04801, https://doi.org/10.1029/ 2011GL050699.

,-- , and — 2013: Impact of asymmetric dynamical processes on the structure and intensity change of two-dimensional hurricane-like annular vortices. J. Atmos. Sci., 70, 559-582, https://doi.org/10.1175/JAS-D-12-0192.1.

$\longrightarrow,-$, and T.-K. Lai, 2018: A possible three-dimensional mechanism for oscillating wobbles in tropical cyclone-like vortices with concentric eyewalls. J. Atmos. Sci., 75, 21572174, https://doi.org/10.1175/JAS-D-18-0005.1.

Mlawer, E. J., S. J. Taubman, P. D. Brown, M. J. Iacono, and S. A. Clough, 1997: Radiative transfer for inhomogeneous atmosphere: RRTM, a validated correlated-k model for the longwave. J. Geophys. Res., 102, 16 663-16 682, https://doi.org/ 10.1029/97JD00237.

Montgomery, M. T., and R. J. Kallenbach, 1997: A theory for vortex Rossby-waves and its application to spiral bands and intensity changes in hurricanes. Quart. J. Roy. Meteor. Soc., 123, 435-465, https://doi.org/10.1002/qj.49712353810.

_- S. F. Abarca, R. K. Smith, C.-C. Wu, and Y.-H. Huang, 2014: Comments on "How does the boundary layer contribute to eyewall replacement cycles in axisymmetric tropical cyclones?"' J. Atmos. Sci., 71, 4682-4691, https://doi.org/ 10.1175/JAS-D-13-0286.1.
Nolan, D. S., and M. T. Montgomery, 2000: The algebraic growth of wavenumber one disturbances in hurricane-like vortices. J. Atmos. Sci., 57, 3514-3538, https://doi.org/10.1175/ 1520-0469(2000)057<3514:TAGOWO>2.0.CO;2.

,$- \ldots$, and L. D. Grasso, 2001: The wavenumber-one instability and trochoidal motion of hurricane-like vortices. J. Atmos. Sci., 58, 3243-3270, https://doi.org/10.1175/1520-0469(2001) 058<3243:TWOIAT $>2.0$.CO;2.

Oda, M., M. Nakanishi, and G. Naito, 2006: Interaction of an asymmetric double vortex and trochoidal motion of a tropical cyclone with the concentric eyewall structure. J. Atmos. Sci., 63, 1069-1081, https://doi.org/10.1175/JAS3670.1.

Pasch, R. J., E. S. Blake, H. D. Cobb III, and D. P. Roberts, 2006: Hurricane Wilma 15-25 October 2005. National Hurricane Center Rep., 27 pp., https://www.nhc.noaa.gov/data/tcr/ AL252005_Wilma.pdf.

Persing, J., and M. T. Montgomery, 2003: Hurricane superintensity. J. Atmos. Sci., 60, 2349-2371, https://doi.org/10.1175/ 1520-0469(2003)060<2349:HS>2.0.CO;2.

Rozoff, C. M., W. H. Schubert, and J. P. Kossin, 2008: Some dynamical aspects of tropical cyclone concentric eyewalls. Quart. J. Roy. Meteor. Soc., 134, 583-593, https://doi.org/10.1002/ qj.237.

_ D. S. Nolan, J. P. Kossin, F. Zhang, and J. Fang, 2012: The roles of an expanding wind field and inertial stability in tropical cyclone secondary eyewall formation. J. Atmos. Sci., 69, 26212643, https://doi.org/10.1175/JAS-D-11-0326.1.

Ryglicki, D. R., and R. E. Hart, 2015: An investigation of centerfinding techniques for tropical cyclones in mesoscale models. J. Appl. Meteor. Climatol., 54, 825-846, https://doi.org/10.1175/ JAMC-D-14-0106.1.

Samsury, C. E., and E. J. Zipser, 1995: Secondary wind maxima in hurricanes: Airflow and relationship to rainband. Mon. Wea. Rev., 123, 3502-3517, https://doi.org/10.1175/1520-0493(1995) $123<3502:$ SWMIHA $>2.0$. CO 2 .

Schecter, D. A., and M. T. Montgomery, 2007: Waves in a cloudy vortex. J. Atmos. Sci., 64, 314-337, https://doi.org/10.1175/ JAS3849.1.

Schubert, W. H., M. T. Montgomery, R. K. Taft, T. A. Gunn, S. R. Fulton, J. P. Kossin, and J. P. Edwards, 1999: Polygonal eyewalls, asymmetric eye contraction, and potential vorticity mixing in hurricanes. J. Atmos. Sci., 56, 1197-1223, https://doi.org/10.1175/1520-0469(1999)056<1197:PEAECA > 2.0.CO;2.

Sitkowski, M., J. P. Kossin, and C. M. Rozoff, 2011: Intensity and structure changes during hurricane eyewall replacement cycles. Mon. Wea. Rev., 139, 3829-3847, https://doi.org/10.1175/ MWR-D-11-00034.1.

Skamarock, W. C., and Coauthors, 2008: A description of the Advanced Research WRF version 3. NCAR Tech. Note NCAR/TN-475+STR, 113 pp., https://doi.org/10.5065/ D68S4MVH.

Stern, D. P., and D. S. Nolan, 2009: Reexamining the vertical structure of tangential winds in tropical cyclones: Observations and theory. J. Atmos. Sci., 66, 3579-3600, https:// doi.org/10.1175/2009JAS2916.1.

_ J. R. Brisbois, and D. S. Nolan, 2014: An expanded dataset of hurricane eyewall sizes and slopes. J. Atmos. Sci., 71, 27472762, https://doi.org/10.1175/JAS-D-13-0302.1.

Terwey, W. D., and M. T. Montgomery, 2008: Secondary eyewall formation in two idealized, full-physics modeled hurricanes. J. Geophys. Res., 113, D12112, https://doi.org/10.1029/ 2007JD008897. 
Thompson, G., P. R. Field, R. M. Rasmussen, and W. D. Hall, 2008: Explicit forecasts of winter precipitation using an improved bulk microphysics scheme. Part II: Implementation of a new snow parameterization. Mon. Wea. Rev., 136, 5095-5115, https://doi.org/10.1175/2008MWR2387.1.

Tsujino, S., K. Tsuboki, and H.-C. Kuo, 2017: Structure and maintenance mechanism of long-lived concentric eyewalls associated with simulated Typhoon Bolaven (2012). J. Atmos. Sci., 74, 3609-3634, https://doi.org/10.1175/JAS-D-16-0236.1.

Wang, H., C.-C. Wu, and Y. Wang, 2016: Secondary eyewall formation in an idealized tropical cyclone simulation: Balanced and unbalanced dynamics. J. Atmos. Sci., 73, 3911-3930, https:// doi.org/10.1175/JAS-D-15-0146.1.

Willoughby, H. E., 1988: The dynamics of the tropical cyclone core. Aust. Meteor. Mag., 36, 183-191.

— J. A. Clos, and M. G. Shoreibah, 1982: Concentric eye walls, secondary wind maxima, and the evolution of the hurricane vortex. J. Atmos. Sci., 39, 395-411, https://doi.org/10.1175/ 1520-0469(1982)039<0395:CEWSWM>2.0.CO;2.

_ J. M. Masters, and C. W. Landsea, 1989: A record minimum sea level pressure observed in Hurricane Gilbert. Mon. Wea. Rev., 117, 2824-2828, https://doi.org/10.1175/1520-0493(1989) $117<2824$ :ARMSLP $>2.0$. CO;2.

Yang, Y.-T., H.-C. Kuo, E. A. Hendricks, and M. S. Peng, 2013: Structural and intensity changes of concentric eyewall typhoons in the western North Pacific basin. Mon. Wea. Rev., 141, 26322648, https://doi.org/10.1175/MWR-D-12-00251.1.

Zhang, F., D. Tao, Y. Q. Sun, and J. D. Kepert, 2017: Dynamics and predictability of secondary eyewall formation in sheared tropical cyclones. J. Adv. Model. Earth Syst., 9, 89-112, https://doi.org/ 10.1002/2016MS000729.

Zhang, G., and W. Perrie, 2018: Effects of asymmetric secondary eyewall on tropical cyclone evolution in Hurricane Ike (2008). Geophys. Res. Lett., 45, 1676-1683, https://doi.org/10.1002/ 2017GL076988.

Zhang, J. A., R. F. Rogers, D. S. Nolan, and F. D. Marks Jr., 2011: On the characteristic height scales of the hurricane boundary layer. Mon. Wea. Rev., 139, 2523-2535, https://doi.org/10.1175/ MWR-D-10-05017.1.

Zhao, K., Q. Lin, W.-C. Lee, Y. Q. Sun, and F. Zhang, 2016: Doppler radar analysis of triple eyewalls in Typhoon Usagi (2013). Bull. Amer. Meteor. Soc., 97, 25-30, https://doi.org/ 10.1175/BAMS-D-15-00029.1.

Zhou, X., and B. Wang, 2011: Mechanism of concentric eyewall replacement cycles and associated intensity change. J. Atmos. Sci., 68, 972-988, https://doi.org/10.1175/2011JAS3575.1.

Zhu, Z., and P. Zhu, 2014: The role of outer rainband convection in governing the eyewall replacement cycle in numerical simulations of tropical cyclones. J. Geophys. Res. Atmos., 119, 8049-8072, https://doi.org/10.1002/2014JD021899. 